\title{
Detection and clinical significance of circulating tumor cells in colorectal cancer
}

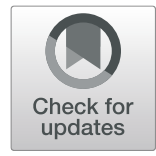

\author{
Miao Jiang ${ }^{1 \dagger}$, Shuiling Jin ${ }^{1 \dagger}$, Jinming Han ${ }^{1}$, Tong Li ${ }^{2}$, Jianxiang Shi ${ }^{2,3}$, Qian Zhong ${ }^{1}$, Wen $\mathrm{Li}^{1}$, Wenxue Tang ${ }^{4 *}$, \\ Qinqin Huang ${ }^{5^{*}}$ and Hong Zong ${ }^{1 *}$ (D)
}

\begin{abstract}
Histopathological examination (biopsy) is the "gold standard" for the diagnosis of colorectal cancer (CRC). However, biopsy is an invasive method, and due to the temporal and spatial heterogeneity of the tumor, a single biopsy cannot reveal the comprehensive biological characteristics and dynamic changes of the tumor. Therefore, there is a need for new biomarkers to improve CRC diagnosis and to monitor and treat CRC patients. Numerous studies have shown that "liquid biopsy" is a promising minimally invasive method for early CRC detection. A liquid biopsy mainly samples circulating tumor cells (CTCs), circulating tumor DNA (ctDNA), microRNA (miRNA) and extracellular vesicles (EVs). CTCs are malignant cells that are shed from the primary tumors and/or metastases into the peripheral circulation. CTCS carry information on both primary tumors and metastases that can reflect dynamic changes in tumors in a timely manner. As a promising biomarker, CTCs can be used for early disease detection, treatment response and disease progression evaluation, disease mechanism elucidation, and therapeutic target identification for drug development. This review will discuss currently available technologies for plasma CTC isolation and detection, their utility in the management of CRC patients and future research directions.
\end{abstract}

Keywords: Colorectal Cancer, Circulating tumor cell, Cellular heterogeneity, Precision medicine, Separation

\section{Introduction}

Colorectal cancer (CRC) is the fourth deadliest cancer in the word, with almost one million deaths annually [1]. In recent years, its morbidity and mortality have increased due to delayed diagnosis and limited treatment options [2]. Early detection and precision treatment are keys to improving the prognosis of CRC patients [3]. In the past, the definite diagnosis of CRC mainly relied on biopsy-based techniques [4]. One single static biopsy

\footnotetext{
* Correspondence: twx@zzu.edu.cn; qqhuang@zzu.edu.cn; fcczongh@zzu.edu.cn

${ }^{+}$Miao Jiang and Shuiling Jin are joint first authors, with Miao Jiang in the first position.

${ }^{1}$ Department of Oncology, the First Affiliated Hospital of Zhengzhou University, NO.1 Eastern Jianshe Road, Zhengzhou 450052, Henan, China ${ }^{5}$ Academy of medical science, Zhengzhou University, Zhengzhou 450052, Henan, China

${ }^{4}$ Departments of Otolaryngology, The Second Affiliated Hospital of Zhengzhou University, Zhengzhou 450000, Henan, China

Full list of author information is available at the end of the article
}

specimen cannot reflect the characteristics of the tumor in real time. Therefore, there is an urgent need for a safer and more real-time method to obtain comprehensive and dynamic information that can reflect the development and treatment response of cancer to supplement or replace solid tissue biopsies.

"Liquid biopsy" has attracted widespread attention from researchers [5-7]. Liquid biopsy refers to the analysis of biomarkers including circulating tumor cells (CTCs), circulating tumor DNA (ctDNA), microRNA (miRNA) and extracellular vesicles (EVs) in peripheral blood or other body fluids [8] This method can overcome the limitations of solid biopsy and reflect the heterogeneous information of the tumor in real time.

CTCs are epithelial cancer cells that have the ability to move, migrate and invade blood vessels after epithelial-mesenchymal transition (EMT) and are considered the main cause of tumor metastasis in vivo

\section{BMC}

(C) The Author(s). 2021 Open Access This article is licensed under a Creative Commons Attribution 4.0 International License, which permits use, sharing, adaptation, distribution and reproduction in any medium or format, as long as you give appropriate credit to the original author(s) and the source, provide a link to the Creative Commons licence, and indicate if changes were made. The images or other third party material in this article are included in the article's Creative Commons licence, unless indicated otherwise in a credit line to the material. If material is not included in the article's Creative Commons licence and your intended use is not permitted by statutory regulation or exceeds the permitted use, you will need to obtain permission directly from the copyright holder. To view a copy of this licence, visit http://creativecommons.org/licenses/by/4.0/. The Creative Commons Public Domain Dedication waiver (http://creativecommons.org/publicdomain/zero/1.0/) applies to the data made available in this article, unless otherwise stated in a credit line to the data. 
[9, 10]. Upon reaching an appropriate niche, CTCs undergo mesenchymal-epithelial transition (MET), reacquire stem cell properties and form a new metastatic site [11]. Unlike other cancer biomarkers, CTCs are live tumor cells that carry molecular and biological information about the tumor as a whole, support single cell analysis, and directly reflect the ongoing changes in tumors at all stages [12].

Studies of different cohorts have shown that the quantity of CTCs is valuable for assessing the prognosis of CRC $[13,14]$. CTCs also show application value in early diagnosis and monitoring the dynamic changes $[15,16]$. The phenotypic and molecular characteristics of CTCs can help reveal the mechanism of pathogenesis and metastasis of CRC and identify specific mutations in target genes $[17,18]$. The value of CTCs in CRC is gradually being confirmed; however, the rarity and heterogeneity of CTCs as well as the development of detection and analysis technologies have limited the widespread acceptance and application of CTCs as a new biomarker. In recent years, a variety of new platforms that can detect different subtypes of CTCs with high sensitivity and specificity have been developed. Researchers are continuing to verify the correlations between CTC characteristics and other clinical characteristics. This review will provide an overview of current and emerging techniques of CTC isolation and detection and discuss their potential clinical value.

\section{CTC enrichment and identification}

CTCs exist before tumor lesions can be detected by medical imaging examination [19]. Patients with advanced tumors will typically have only 1 CTC out of $1 \times$ $10^{6}$ neutrophils in $1 \mathrm{~mL}$ of peripheral blood, and the number of CTCs in early-stage patients is even lower [20]. In addition, CTCs are susceptible to apoptosis and inherent fragility. When detached from the original focus, they are prone to undergoing phenotypic changes, and only a few cells can survive [21] (Fig. 1). Liquid biopsy allows repeated sampling and has the potential to assess tumor heterogeneity and the clonal diversity of drug resistance. Such information is of great significance for accurate drug administration and improving patient prognosis. Several systems have emerged to improve the detection efficiency of CTCs, which can be broadly

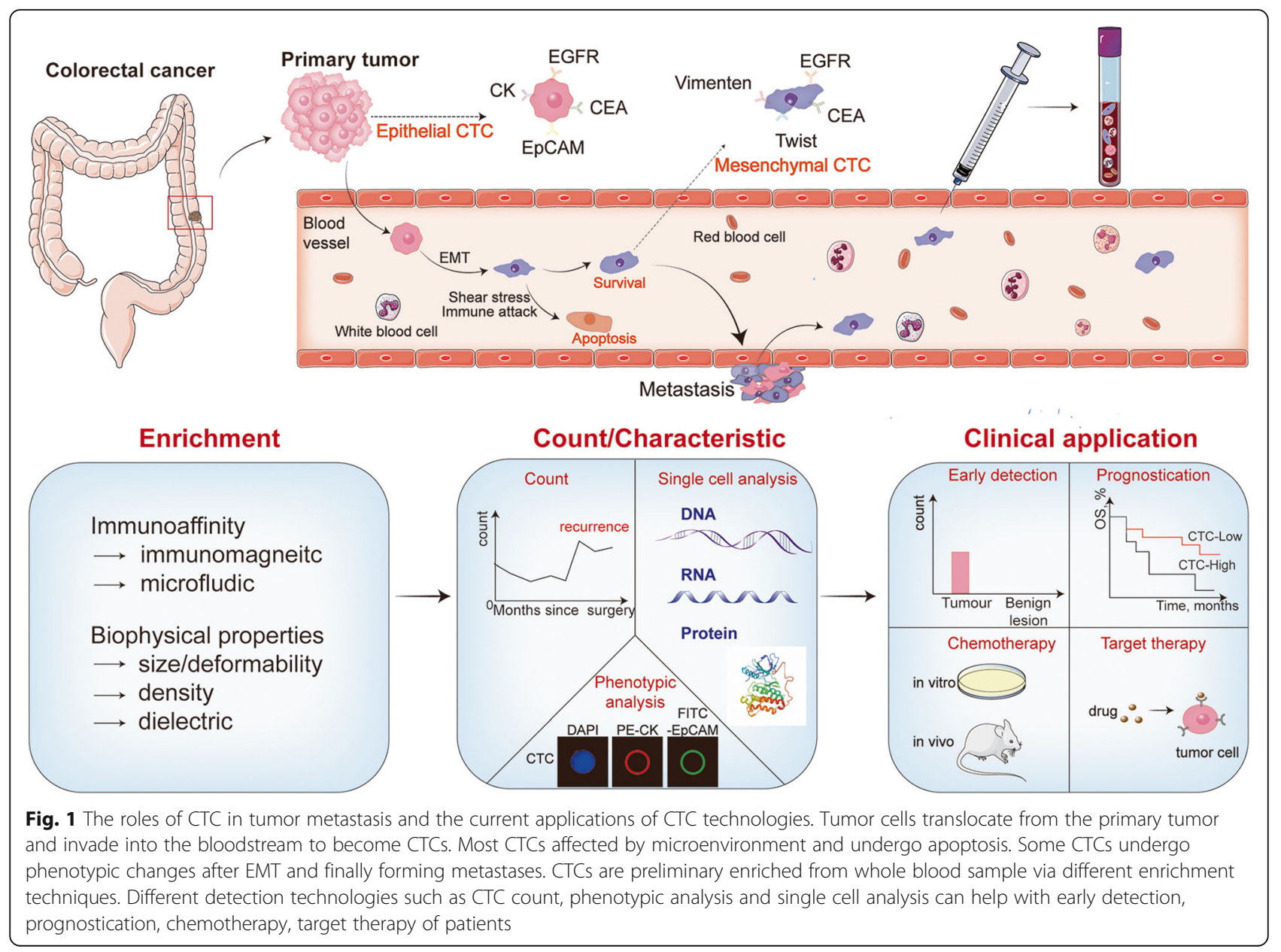




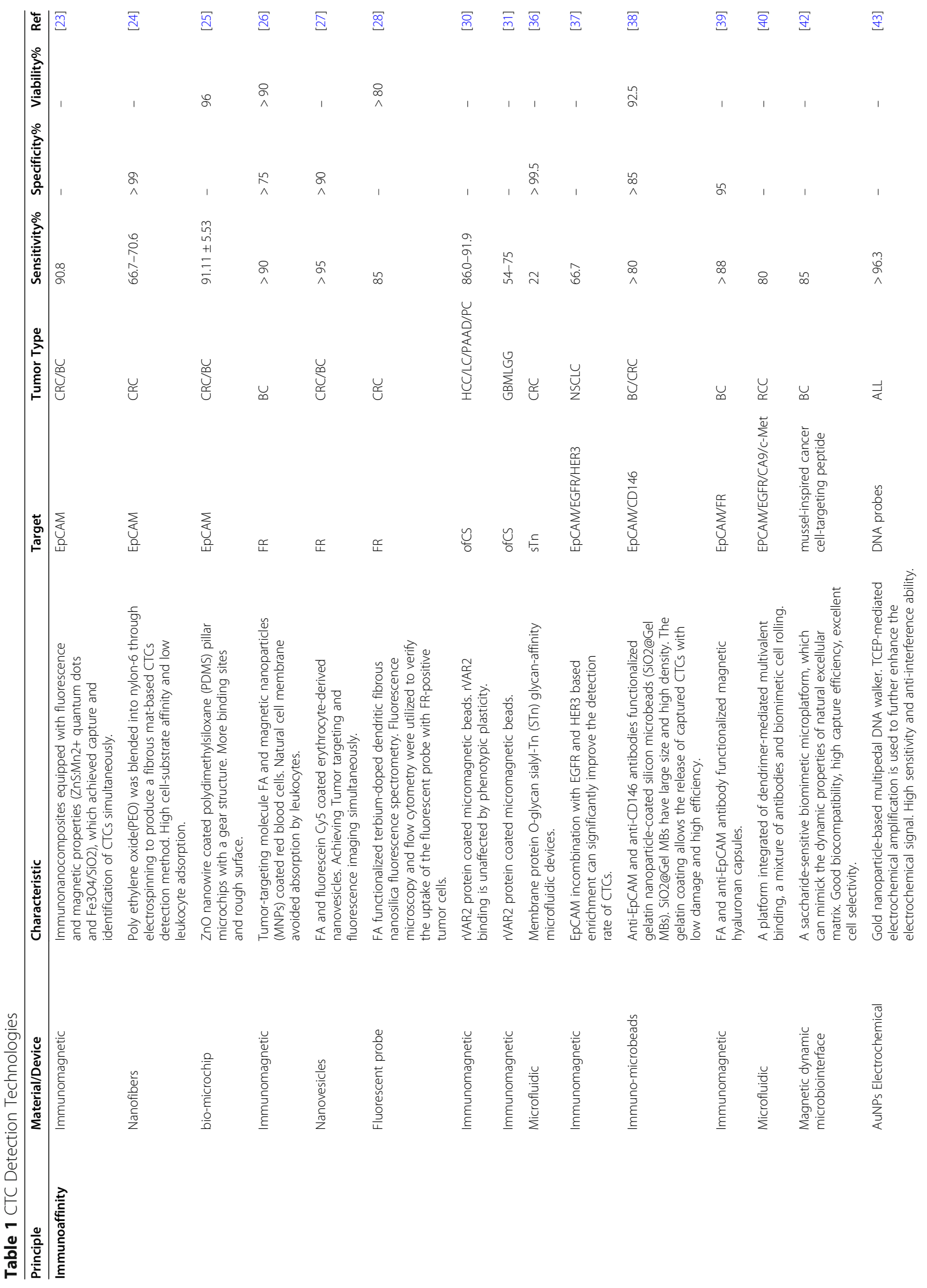


Jiang et al. Biomarker Research

(2021) 9:85

Page 4 of 20

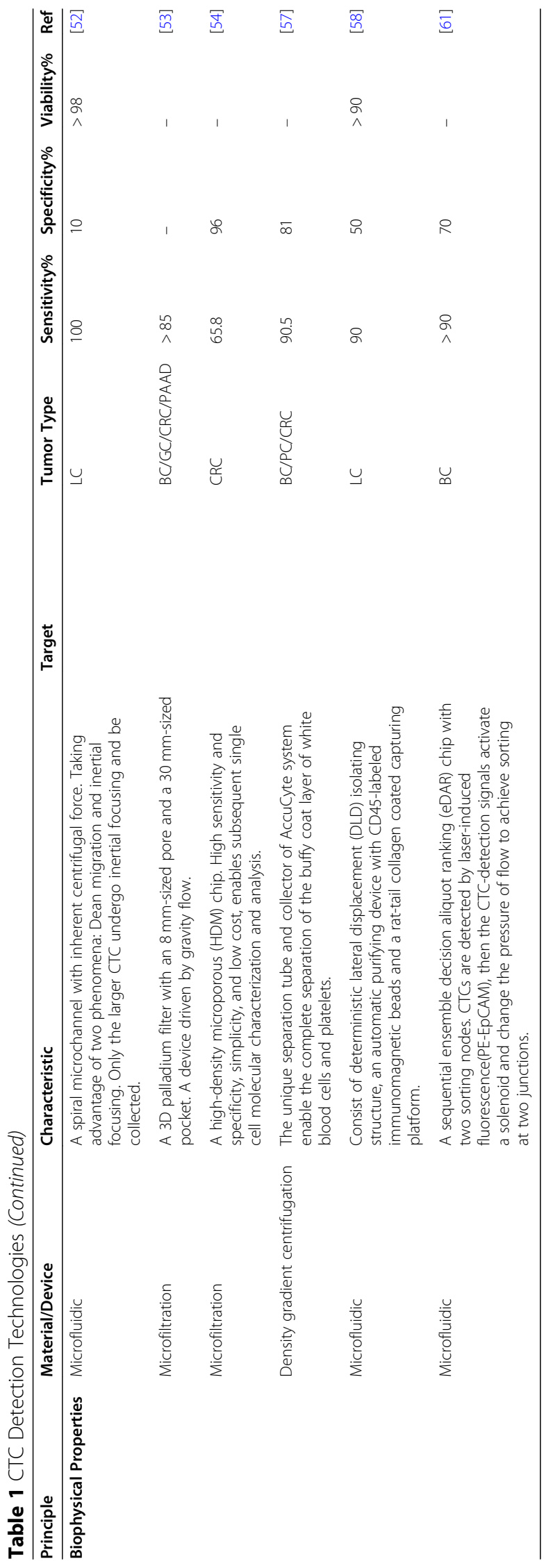


classified into two categories: immunoaffinity-based enrichment and biophysical property-based enrichment (Table 1).

\section{Immunoaffinity}

Immunoaffinity-based CTC enrichment techniques, which use molecular probes such as antibodies, peptide chains, and aptamers, are the most common category. The most widely used immunomagnetic separation methods are positive enrichment based on epithelial cell adhesion molecule (EpCAM) and negative enrichment based on CD45. The most representative system is the CellSearch system, which is the first and only system to meet the standard of the US Food and Drug Administration (FDA). The CellSearch system utilizes ferrofluid beads coated with EpCAM antibodies and then identifies cells using 4',6-diamidino-2-phenylindole (DAPI)-based cell nuclear staining, CD45-allophycocyanin-specific leukocyte negative selection and cytokeratin 8,18,19phycoerythrin-specific epithelial cell positive selection to creat an objective indicator (EpCAM+, DAPI+, CD45-, cytokeratin+) of CTC counts. The CellSearch system has been approved for the diagnosis and prognosis of patients with CRC, breast cancer and prostate cancer [22].

EpCAM, which is representative of the markers used in positive enrichment techniques, is epithelial-specific and is strongly expressed in most cancers. In recent years, a variety of CTC enrichment technologies based on EpCAM have emerged. Cui et al. engineered a fluorescent and magnetic immunonanocomposite $\left(\mathrm{ZnS}: \mathrm{Mn}^{2+}\right.$ quantum dots (QDs) and $\mathrm{Fe}_{3} \mathrm{O}_{4} / \mathrm{SiO}_{2}$ ) modified with an EpCAM antibody for fluorescence identification of CTCs by the fluorescence emitted by $\mathrm{ZnS}: \mathrm{Mn}^{2+}$ QDs during the capture of CTCs. This method omitted the complex steps of traditional three-color fluorescence identification and efficiently reduced cell damage and loss [23. Lee et al. engineered an EpCAM antibody-modified binary-blend fiber [24] (Fig. 2a). By blending polyethylene oxide (PEO) into nylon- 6 by electrospinning, nonspecific binding was reduced while providing a rough nanosubstrate, which facilitated high cell-substrate affinity and low leukocyte adsorption. The blood samples of 23 CRC patients were analyzed, and the results were compared with those of colonoscopic biopsy and the IsoFlux system. They concluded that the new method could be used for the diagnosis of CRC via CTC status. In addition to the properties of the material itself, the surface properties of the material can also be optimized. Cui et al. constructed novel EpCAM antibodyfunctionalized $\mathrm{ZnO}$ nanowire-integrated biomicrochips [25] (Fig. 2b). ZnO nanowire arrays provided a rough surface for gear structure polydimethylsiloxane(G-

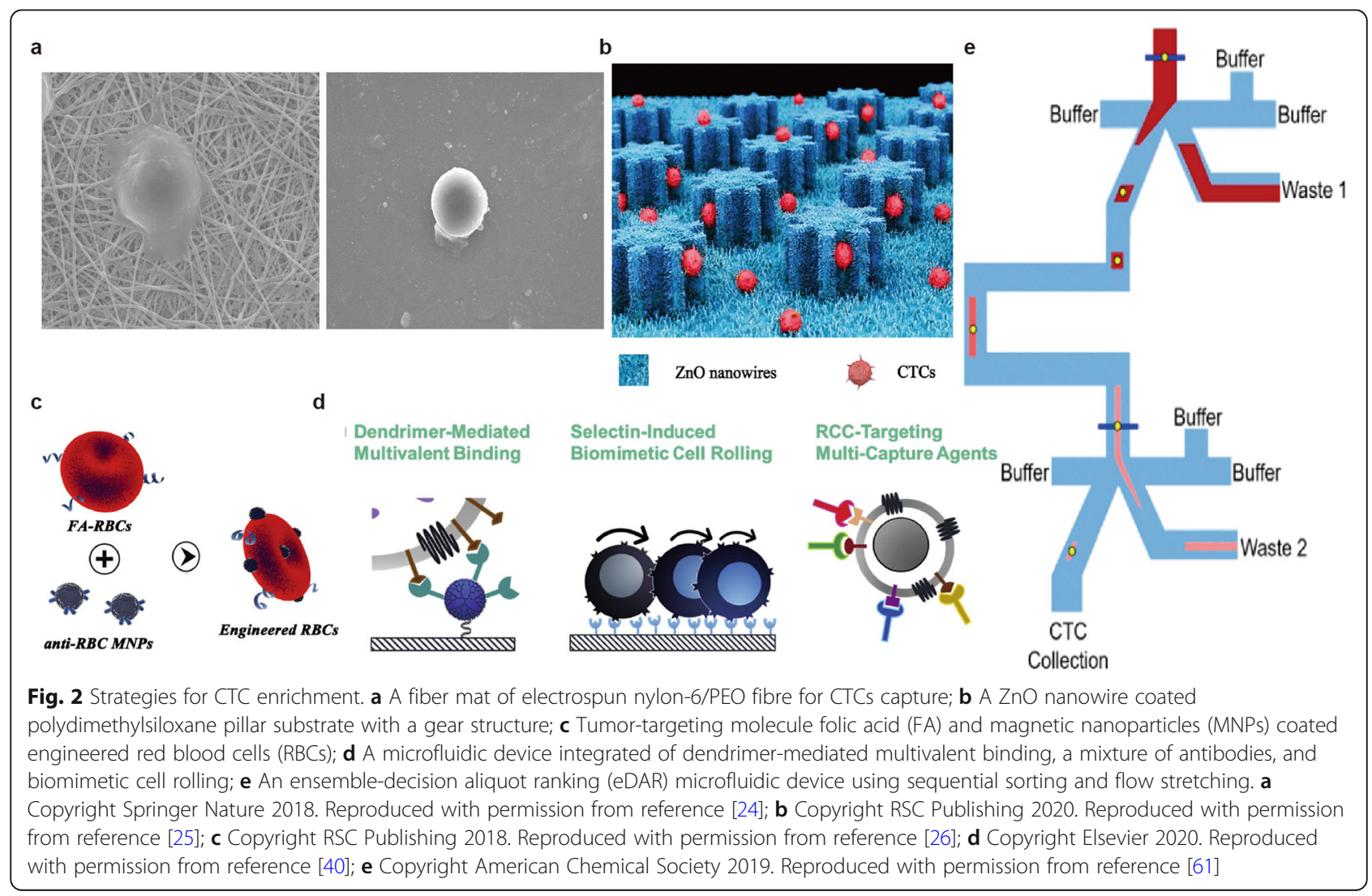


PDMS), while the gear structure provided more binding sites for antibodies and target cancer cells. The microchip achieved a high capture rate $(91.11 \% \pm 5.53 \%)$ and release rate $(>90 \%)$ of CTCs, and the high sensitivity of $\mathrm{ZnO}$ nanowires to $\mathrm{pH}$ enabled the captured CTCs to be released quickly, efficiently, and with minimal damage under weakly acidic conditions.

Metastasis is the major challenge in cancer management. Many studies have suggested that primary tumor cells need to undergo EMT before invading blood vessels and gaining metastatic ability. These cells experience different degrees of EMT and gain several different subtypes, including the epithelial type, mesenchymal type or mixed type, and the expression of EpCAM on the surface of CTCs is downregulated to varying degrees, making it difficult for these heterogeneous cells to be enriched by EpCAM-dependent CTC capture technologies. These tumor cell subsets are of great significance for studying the mechanism of cancer transmission and metastasis. Therefore, researchers have improved many aspects of CTC capture techniques, such as antibodies, biological probes, and the source of blood samples.

Optimizing antibody application strategies, such as searching for different CTC-specific biomarkers, is an effective approach to improve CTC capture efficiency. To meet the demand of rapid division, folate receptors (FRs) are overexpressed on the surface of most tumor cells to absorb folic acid (FA) to promote the biosynthesis of DNA and RNA. Zhu et al. developed FA-coated and magnetic nanoparticle-coated engineered homogeneous red blood cells and obtained a high capture rate (>90\%) and survival rate (>90\%) in artificial whole blood samples; in addition, the purity (>75\%) was much higher than that of EpCAM-based immunomagnetic separation technology (20\%) [26] (Fig. 2c). Complexes in physiological environments can cover the surface of the magnetic beads, affecting their binding to tumor cells. Moreover, nonspecific binding with white blood cells is high, leading to a decline in purity. The immune escape mechanism of natural cells greatly reduces nonspecific binding, showing their potential as highly specific CTC capture materials. Chen et al. engineered an FA modified erythrocyte-derived nanovesicle as a tumor-targeting molecules [27]. They achieved a high capture efficiency (>90\%) in whole blood, while magnetic-activated cell sorting (MACS) reached only $50 \%$. Jafar et al. developed FA-functionalized terbium-doped dendritic fibrous nanosilica and achieved a lower limit of detection of 500 cells/ml for HT29 cancer cells by spectrofluorimetric cytosensing [28]. Oncofetal chondroitin sulfate is specifically expressed in the placenta and in almost all types of tumor cells, indicating that it may be an ideal target. In 2015, a smaller recombinant VAR2CSA protein encompassing ID1-ID2a (rVAR2) was shown to specifically bind to 95\% (106/111) of different phenotypes of human cancer cells with little binding to normal tissue cells [29]. rVAR2 has great potential for CTC-specific capture and targeted drug therapy. In 2018, the same group presented a CTC isolation method based on rVAR2modified micrometer-scale magnetic beads and the IsoFlux System [30]. They confirmed the specificity of rVAR2 for tumor cells, which was independent of cell phenotypes [31]. rVAR2 could potentially be used to target different types of cancer cells and guide precision medicine [32-34]. In 2020, they optimized several parameters of this technology [35], including the size and concentration of the magnetic beads and the recombinant forms and concentration of rVAR2. Compared with the widely used direct CTC capture approach, the indirect capture approach of preincubating cells and antibodies was more effective for rVAR2-based cancer cell retrieval under conditions of low target expression. Their findings may serve as an inspiration for the development and optimization of other bead-based CTC capture technologies. Membrane protein O-glycan sialyl-Tn (STn) antigen is overexpressed in advanced digestive tract tumors such as CRC and gastric cancer but not in normal blood cells and is often associated with tumor metastasis and poor prognosis. Manuel et al. proposed that for the same CRC patient, $\mathrm{STn}^{+}$CTC counts were significantly higher than $\mathrm{EpCAM}^{+}$CTCs counts, and they constructed a glycan affinity-based microfluidic device that improved the separation efficiency of CTCs in the peripheral blood of CRC and bladder cancer patients [36].

However, the application of new markers also faces the obstacle of the heterogeneity of ligand expression levels. Exploiting synergism between antibodies may improve the sensitivity of CTC detection technologies. In a cohort of 45 non-small cell lung cancer (NSCLC) patients [37], the positive rate of CellSearch was only 33\%, and the enrichment based on EGFR/HER3 was 37.8\%. When both antibodies were combined, the positive rate (66.7\%) increased significantly. Huang et al. functionalized gelatin nanoparticle-coated silicon microbeads $\left(\mathrm{SiO}_{2} @ G e l \mathrm{MBs}\right)$ with anti-EpCAM and anti-CD146 antibodies to enhance the capture rate of mesenchymal CTCs with low EpCAM expression [38]. Compared with the traditional EpCAM-based method, higher capture efficiency $(>80 \%)$ and higher purity $(>85 \%)$ were obtained. $\mathrm{Ma}$ et al. constructed multitargeting magnetic capsules (TMCs) with an anti-EpCAM antibody and FA and captured and isolated more than $88 \%$ of tumor cells in 15 min of incubation, with high specificity [39]. Jiyoon et al. developed a novel capture platform utilizing four antibodies against renal cell carcinoma (RCC) CTCs, including EpCAM, carbonic anhydrase IX (CA9), epidermal growth factor receptor (EGFR) and hepatocyte growth factor receptor (c-Met). This platform increased the 
capture rate of RCC cells to up to $80 \%$ and was significantly superior to other capture surfaces functionalized with a single antibody $(\leq 60 \%)$ [40] (Fig. 2d). However, not all strategies can improve capture performance. Tim $\mathrm{N}$ et al. used two methods to capture CTCs: the standard EpCAM CellSearch kit (unicapture) and the combined ferrofluid capture of EpCAM plus HER2-, EGFRand MUC-1-specific antibodies (quadcapture). However, the quadcapture ferrofluid reagent failed to significantly improve the CTC capture efficacy [41]. It is necessary to explore and verify different antibody combination strategies.

In addition to specific antibodies, a variety of molecular probes can be used to target tumor cells. Tian et al. constructed a magnetic dynamic microbiointerface with a biofeedback mechanism that mimicked the dynamic properties of the natural extracellular matrix (ECM) and reversible ligand-receptor interactions [42]. This saccharide-sensitive biomimetic microplatform could release CTCs via a molecular exchange mechanism with low damage and achieved high cell capture (85\%) and release (93\%) efficiencies. Peng et al. developed a gold nanoparticle-based multiple DNA walker for the ultrasensitive detection of CTCs for the first time (capture rate $>96.3 \%$ ) [43]. By modifying a number of walker strands on gold nanoparticles (AuNPs), the integrated aptamer sequence can specifically bind to the transmembrane receptor protein of CTCs. During DNA walking, several tracks hybridized with the electrode simultaneously for subsequent nicking endonuclease-catalyzed cleavage. Then, TCEP-mediated electrochemical amplification was employed to obtain an enhanced electrochemical signal. The lower limit of detection was 1 cell/ $\mathrm{ml}$. The outstanding resolution of CTCs in blood samples suggests their potential application prospects in early diagnosis of cancer.

Most in vitro CTC enrichment technologies rely on small sample volumes $(5-10 \mathrm{ml})$, and researchers strive to improve the sensitivity. Due to the scarcity and uneven distribution of CTCs, the usual sources and volume of peripheral blood samples limit the improvement of the technology. In vivo CTC detection technology has aroused the interest of researchers. This kind of technology can continuously process large volumes of blood to simultaneously obtain more CTCs and enable dynamic monitoring. At present, there are two main types of in vivo detection technologies. One representative technology is CellCollector (GILUPI CellCollector, GILUPI, Potsdam, Germany) [44]. A wire modified by an EpCAM antibody is placed into the vein of the arm for $30 \mathrm{~min}$, and then the tumor cells on the steel wire are identified under a fluorescence microscope for early cancer diagnosis. However, it can only be used for CTC counting, and no further analysis can be carried out. Moreover, the specific blood volume varies between patients and at different time points for the same patient, which makes it difficult to further evaluate characteristics such as the change in CTC count during treatment. Another kind of method is in vivo imaging flow cytometry [45]. By visualizing of tumor cells and blood vessels in advance, the interactions between cells circulating in the blood can be directly observed. This technique has great potential for observing the interactions among targeted drugs, immune cells and tumor cells. However, CTCs cannot be directly obtained for downstream analysis, and this kind of method may affect the physiological state of the patient. Tang et al. constructed an extracorporeal circulation system based on an in vivo microfluidic chip detection system (IV-chip-system) [46]. Venous blood is continuously drained into the extracorporeal circulation system to detect and monitor CTCs, and then the blood is returned back to the body directly. This method enables the analysis of a large amount of blood with low damage to the body. At present, it has only been tested in mouse models, and more research is needed to evaluate the feasibility of this technique in the human body.

The identification of CTCs after enrichment still depends on epithelial biomarkers. The damage and loss of CTCs in the complex fluorescence labeling process can be avoided by developing a post-enrichment identification method. Li et al. constructed a hydrogen peroxideresponsive nanoprobe [47]. Tumor cells have an exuberant metabolism and high levels of endogenous hydrogen peroxide, and the probe can quickly respond to high levels of intracellular hydrogen peroxide and emit fluorescence signals. Besides increasing the volume of blood samples, the source of blood samples can be expanded to increase the number of CTCs detected. Researchers found that more CTCs were detected in tumor-drained venous blood or mesenteric-drained venous blood than in peripheral blood [48-50]. CTCs were detected before the liver first-pass effect to reduce the negative effect on the number of CTCs in peripheral blood. Compared with the conventional detection of peripheral blood, the detection rate and capture rate were higher.

\section{Biophysical properties}

Biophysical property-based enrichment technology mainly exploits the differences in size, deformability, density and dielectric properties between CTCs and blood cells, which eliminates need to address the heterogeneity of cell surface antigen expression. In addition, the isolated CTCs are more suitable for downstream analysis without antibody labeling or special treatment [51]. CTCs of various phenotypes are captured almost indiscriminately. The comprehensive analysis of different phenotypes of CTCs can facilitate the determination of 
the value of CTCs in guiding treatment and predicting prognosis.

The size-based devices isolate CTCs mainly utilize the physical and mechanical differences between CTCs (10$20 \mu \mathrm{m}$ ) and other blood components (red blood cells, $8 \mu \mathrm{m}$; leukocytes, 7-12 $\mu \mathrm{m}$; better deformability). Since there is some degree of overlap in the size of CTCs and leukocytes, it's crucial to choose an appropriate threshold. A size of $8 \mu \mathrm{m}$ is usually considered the appropriate cutoff value. Taking advantage of the size difference between CTCs and hematologic cells, Han et al. constructed a spiral microchannel with a recovery rate of more than $85 \%$ of a breast cancer cell line (MCF-7) using centrifugal force [52]. Under the influence of Dean drug forces, all the cells migrate from the outer channel to the inner channel along the Dean vortex. The smaller cells will eventually return to the outer wall and be discarded, while the larger cells will experience additional strong inertial lift forces and focus along the inner wall to be collected. Akiko et al. developed a 3-dimensional (3D) palladium filter driven by gravity flow that can recover CTCs from whole blood with a recovery rate of more than 85\% [53]. Bo Young Oh et al. used a highdensity microporous (HDM) chip to detect CTCs in 76 CRC patients [54]. Whole blood samples were pretreated and filtered with the HDM chip, and the positive rate of CTCs was $65.8 \%$. Anil et al. constructed an electrical double layer (EDL)-gated $\mathrm{AlGaN} / \mathrm{GaN}$ high electron mobility transistor (HEMT) biosensor array that utilizes the response to cell transmembrane potential to detect and count tumor cells and can also be used to monitor the dynamic transformation of fine membrane potential [55]. Parham et al. utilized the specific differential impedance curve generated when CTCs are deformed through an $8 \mu \mathrm{m}$ channel. In addition to identification and counting, the accuracy of identifying the origin of cancer cells was more than $90 \%$ [56].

The density of CTCs is intermediated between that of plasma and red blood cells but within the range of leukocyte density. By taking advantages of the different sedimentation coefficient in the density medium (such as Ficoll-Paque medium) and density-based gradient centrifugation, cells of different densities are distributed in layers in the separation solution to realize the separation of CTCs. For example, the principle of the AccuCyte system is to use the specific density of CTCs, but unlike other density-based separation methods, its unique separation tube and collector completely separate the buffy coat layer of white blood cells and platelets, avoiding the loss of a large number of CTCs [57]. After separation, CTCs are identified and characterized by a CyteFinder system. The average recovery rate for suspended tumor cells was $90.5 \%$. Verification in a clinical cohort of CRC revealed that the CTC counting ability of the system was better than that of CellSearch. However, there is some overlap of physical properties between leukocytes and CTCs, which will lead to poor specificity and the loss of some CTCs.

CTC enrichment techniques based on immune affinity and biophysical properties have their own advantages and limitations. The emergence of integrated microfluidic devices combines the advantages of both and improves the sensitivity and specificity of monitoring and detection. In addition, the enrichment, identification and analysis of CTCs can be realized on one platform, which saves time and reduces the damage and loss of CTCs during the transfer process. Jiang et al. constructed an integrated microfluidic device consisting of a deterministic lateral displacement (DLD)-isolating structure, an automatic purifying device with CD45-labeled immunomagnetic beads and a rat tail collagen-coated capturing platform, which can capture cancer cells with high throughput [58]. Taking advantage of fluid characteristics, negative enrichment of multistep purification achieved a 90\% capture rate, $50 \%$ purity and over $90 \%$ survival rate. The positive rate in clinical patients was $83.3 \%$. There was no statistical difference in the capture rate between this system and CellSearch, but this system has the advantages of blood volume, time and cost. The same research group then optimized the staining and separation steps of the device and the obtained CTCs could be analyzed at singlecell resolution [59]. They detected six gene mutations, indicating that this device can effectively obtain CTCs with little damage to their activity and can be used for downstream analysis. Eleanor $S$ et al. designed a sequential ensemble decision aliquot ranking (eDAR) chip with two sorting nodes and realized the capture of CTCs with high purity relative to white blood cells in whole blood by making use of fluid characteristics. In previous studies, they proved that the eDAR platform could capture both $\mathrm{EPCAM}^{\text {high }}$ and $\mathrm{EPCAM}^{\text {low }}$ CTCs simultaneously, and the capture efficiency $(>90 \%)$ was relatively high [60]. After further improvements, cells could also be collected and transferred out of the chip, and one single CTC could be collected in a porous plate. The average number of contaminating leukocytes in each well was less than one, which enabled eDAR to realize the downstream analysis of a single CTC [61] (Fig. 2e). Chen et al. constructed an EpCAM antibodyfunctionalized microfluidic device by using 3D printing technology [62]. The interaction between CTCs and antibodies in the microfluidic channel was effectively improved by optimizing parameters such as intracavity surface area and fluid flow. Both $\mathrm{EPCAM}^{+}$cells and EPCA $\mathrm{M}^{-}$cells were successfully captured. 


\section{Overview of the clinical application value of CTCs} CTCs entering circulation are heterogeneous groups from different tumor lesions and their phenotypic and molecular characteristics differ depending on the microenvironment and treatment. The detection and analysis of CTCs is a new approach for studying the heterogeneity of systemic tumors and promoting the development of precision medicine. The merts and demerts of CTCbased methods, clinical methods and other biomarkers were summarized in (Table 2).

\section{CTC quantification}

Evaluating the clinical and histopathological features of patients has significantly enhanced prognosis and treatment decisions for several cancer subtypes, but there is still much space for improvement. The quantification of serum prostate-specific antigen (PSA) has always been an important indicator of drug efficacy and prognosis in prostate cancer. A large docetaxel-based prospective cohort study of 212 prostate cancer patients found that adding day 0 CTC counts to baseline PSA and other covariates improved the survival prediction accuracy from 8 to $10 \%$. After 3 weeks of treatment, the median overall survival (OS) of patients with increased CTC counts was more reliable than the PSA level for patient prognosis [63]. Satelli et al. found a significant correlation between CTC count and castration resistance in a cohort of 48 metastatic prostate cancer patients, whereas no significant correlation with the quantitative change in PSA was observed [64]. Transcriptional activity of CTCs collected before treatment in patients with different stages of prostate cancer was quantified digitally and patients with high CTC scores had worse responses to treatment and were prone to metastasis [65].

Similarly, in a meta-analysis of the data of 1944 patients with breast cancer, the researchers established a clinicopathological predictive model that includes CTC counts. The Likelihood ratio (LR) $\chi^{2}$ statistical analysis showed that this approach improved the accuracy of prognosis, whereas serum tumor markers (CEA and CA15-3) did not improve prognostic value [66]. An analysis of data from a large cohort of early breast cancer patients showed that patients who received radiotherapy after breast-conserving surgery had significantly longer local recurrence-free survival, disease-free survival (DFS), and OS than patients with CTCs detected before adjuvant therapy. This study suggested for the first time that CTC status can act as a predictive marker of local treatment benefits in patients with early breast cancer [67]. A randomized clinical trial (BRE12-158) analyzed the correlation between postoperative CTC status and follow-up prognosis in 123 patients with early triplenegative breast cancer. With distant disease-free survival (DDFS), DFS and OS as the primary outcome measures, the prognosis of CTC positive patients was significantly worse [68]. However, the above studies were limited by the CTC detection technology, follow-up time, patients' information and other factors, and there remains an unmet need for prospective clinical trials to verify the value of CTCS. It is well known that the EMT process is closely related to metastasis. The proportion of mesenchymal CTCs combined with the total number of CTCs can provide more prognostic information. Guan et al. analyzed patients with metastatic breast cancer (MBC) and determined two thresholds: a total CTC count $\geq 10$ / $5 \mathrm{ml}$ and proportion of mesenchymal CTCs $>10.7 \%$. The median progression-free survival (PFS) of those who meet the above conditions was significantly shorter [69]. CTC counts may serve as an early indicator for selecting treatment options. For hormone receptor-positive $(\mathrm{HR}+)$ MBC patients treated with letrozole plus bevacizumab $($ Let+Bev) or letrozole alone as a first-line treatment regimen, only patients who were baseline CTC positive showed a potential OS benefit from the Let+Bev regime [70]. If confirmed, CTCs may be an effective marker for predicting benefits in $\mathrm{HR}+\mathrm{MBC}$ patients treated with Bev or other targeted drugs.

The predictive value of CTCs in NSCLC has not been officially confirmed. However, according to current information, its potential as a predictive factor cannot be ignored. Chemi et al. detected CTCs in pulmonary vein blood collected during surgery (CellSearch, positive rate was $48 \%$ ). The comparision of the genomic profiles of CTCs, primary lesions and metastases suggested that CTCs spread to the pulmonary vein during surgery and form metastatic foci; thus, CTCs derived from the pulmonary vein during surgery can serve as an independent predictor of recurrence [71]. Pulmonary venous blood can only be collected once, which limits the ability of CTCs to dynamically monitor tumors. However, compared with radial artery blood, the positive rate and CTC count were higher, suggesting the presence of central clearance [72]. According to the patient's condition and the mode of treatment, blood samples from different sources can provide more meaningful information for the clinic. A prospective clinical study of 93 patients with early-stage NSCLC treated with stereotactic body radiation therapy showed that high levels of pretreatment CTCs $(\geq 5 \mathrm{CTCs} / \mathrm{ml})$ and persistent post-treatment CTCs were significantly associated with an increased risk of off-site recurrence [73]. This finding also suggests that CTC quantification has the potential to identify patients at higher risk of recurrence. Preliminary evidence indicates that the status and count of CTCs have potential for early diagnosis, staging and prognosis, which is of great significance for the timely adjustment and optimization of treatment $[74,75]$. 


\section{Molecular characterization of CTCs}

CTCs carry the molecular characteristics of primary tumor cells. A comprehensive analysis of the information shared by the CTC population can be used to judge the origin and diagnosis of tumors. CTCs also carry acquired mutations different from those found at the sites of exfoliated, which can be used to monitor tumor drug resistance and the acquisition of metastatic potential [76]. Combining these two types of information can provide unique insights on tumor heterogeneity at the level of the tumor genome, transcriptome, proteome and function $[77,78]$.

In recent years, immunotherapy has developed rapidly and brought new hope to patients previously treated with chemotherapy for refractory tumors. Immunotherapy mainly uses immune checkpoint inhibitors targeting programmed cell death protein-1/ programmed cell death-ligand 1 (PD-1/PD-L1). However, patients who can benefit from immunotherapy are limited. In addition, acquired drug resistance easily occurs during treatment. The molecular characteristics of CTCs can reveal cell-cell interactions and suggest available therapeutic targets, thus potentially allowing the identification of patients who can benefit from immunotherapy. The detection of CTC immune checkpoints (e.g., PD-L1 and CD47) can predict prognosis and treatment efficacy for patients receiving immunosuppressive drugs [79-81]. However, the predictive value varies with different tumor stages, suggesting that the innate immunity and acquired immune evasion mechanism of CTCs may be related to their metastatic potential. After preliminary verification in lung cancer, breast cancer and other cancers, CTCs were suggested to have the potential to become an independent prognostic biomarker of immunotherapy. There were both consistencies and differences in the expression of PD-L1 in tumor tissue and CTCs, and parallel evaluation of peripheral and local immunity provided more comprehensive information [82, 83]. In addition, the expression of the nuclear androgen receptor splice variant 7 (AR-V7) protein on CTCs is related to androgen receptor-targeted drug resistance, which can guide drug selection for metastatic castrationresistant prostate cancer (mCRPC) patients [84, 85]. The expression of specific proteins on CTCs could be used to predict drug sensitivity and select appropriate drugs.

The most appropriate targeted therapy regimen could be selected according to the genotypic changes of CTCs, and the efficacy could be evaluated over time. For example, the anaplastic lymphoma kinase (ALK) gene fusion mutation is a common strong carcinogenic driver gene in NSCLC, second only to EGFR. ALK inhibitors can effectively prolong the survival time of NSCLC patients with positive ALK gene fusion mutations and address the lack of drug availability after drug resistance.
However, due to ALK rearrangement, patients will develop resistance to ALK inhibitors. Single CTC sequencing can be used to identify drug resistance mutations in patients with ALK rearrangement and evaluate the mechanism of heterogeneous drug resistance to facilitate clinical decision-making [86]. Breast cancer patients with HER2+ CTCs may benefit from corresponding targeted therapies [87].

With the development of next-generation sequencing (NGS) technology, the application of CTCs in highthroughput molecular diagnosis has become possible. Analyzing and comparing the consistency between gene mutations in CTCs and primary lesions/metastases [88, 89], combined with the clinical characteristics and treatment status of patients, will be helpful to understand the immune escape mechanism, drug resistance mechanism, and invasive ability acquisition mechanism [90] and find new signaling pathways.

\section{Clinical implications of CTCs for CRC Diagnosis and assessment of prognosis}

Existing data have clearly demonstrated the clinical significance of CTCs in different stages of CRC. However, further investigations are needed to demonstrate whether CTCs can provide the expected value in clinical applications and guide clinical decision-making compared with tumor biomarkers that have been verified and widely accepted (Table 3).

The quantification of CTCs can be used for the early diagnosis of CRC. CTC counts differ significantly between patients with colorectal polyps and those with nonmetastatic CRC. These differences hold potential for disease screening and the diagnosis of suspected malignant lesions, including even indicating the anatomical location and differentiation of tumors [91]. A prospective clinical study presented at the 2018 American Society of Clinical Oncology (ASCO) conference used the CellMax biomimetic platform for early CRC screening [92]. A total of 620 samples (182 healthy controls, 111 patients with precancerous lesions, and 327 patients with stage I-IV CRC) were enrolled. The results of CTC detection were compared with the results of routine clinical diagnostic methods such as colonoscopy and biopsy, and the overall accuracy was $88 \%$. The false positive rate reported in the healthy control group was low (3.3\%), and the false negative rate of cancer patients was $16 \%$, which was within the acceptable range. Yasmine et al. reported that the metabolic profiles of different types of cells are different. Living single-cell mass spectrometry (LSC-MS) can be used to distinguish lymphocytes and CTCs derived from the same patient, as well as CTCs from different cancer types [93] (Fig. 3a). They found that eicosanoids, acyl carnitine metabolites, and sterol lipids were specifically increased in CRC cells. The analysis of the 


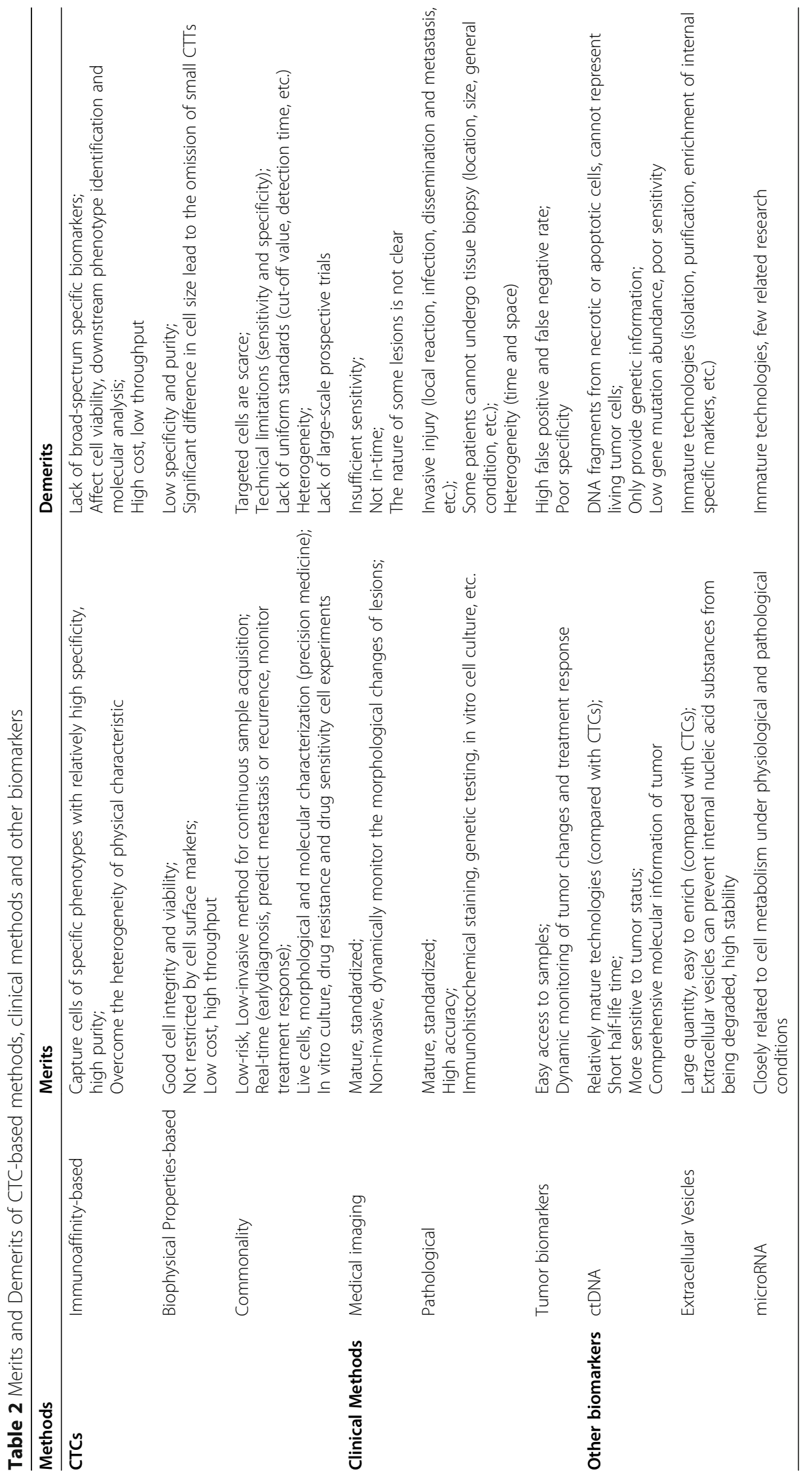




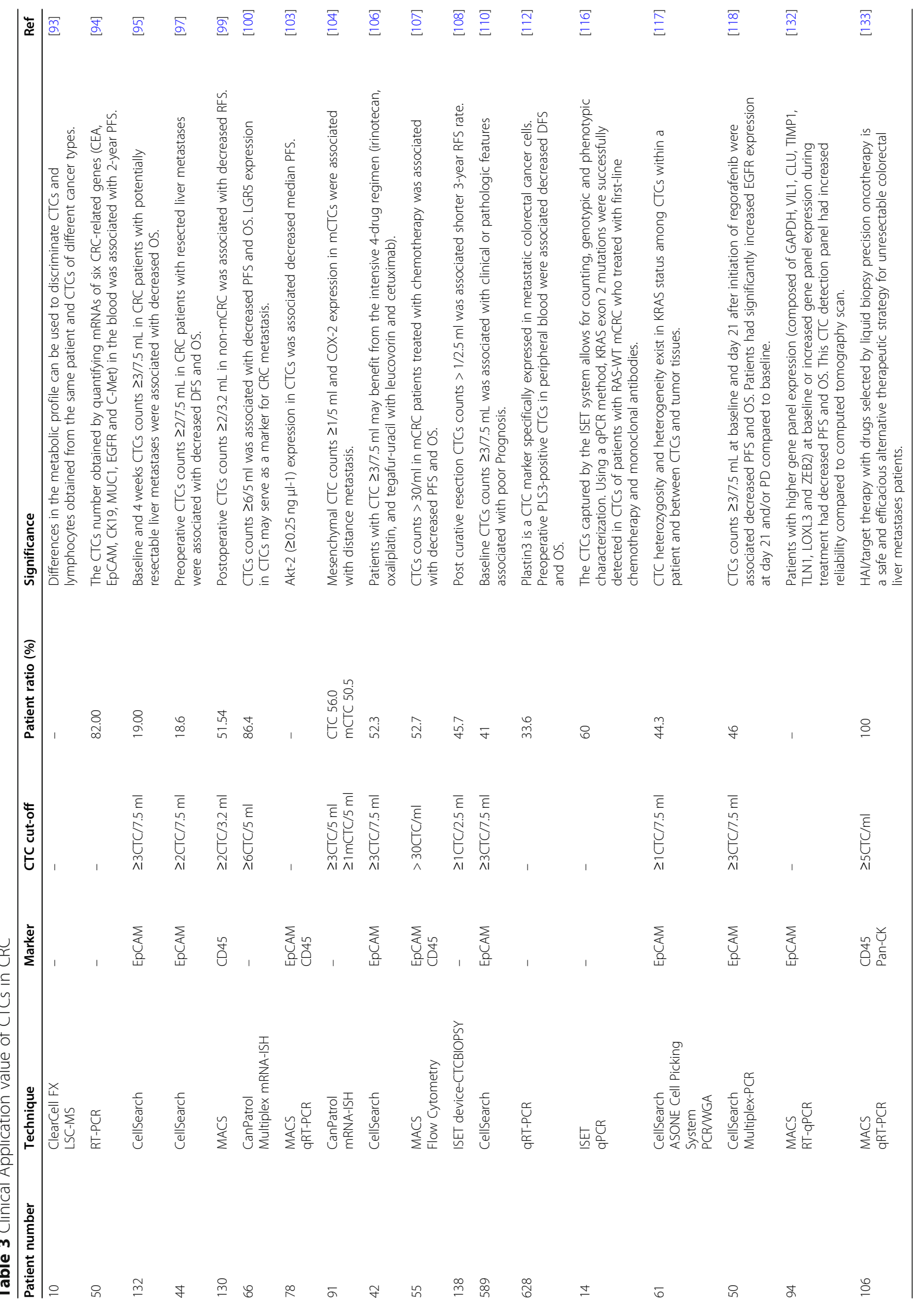




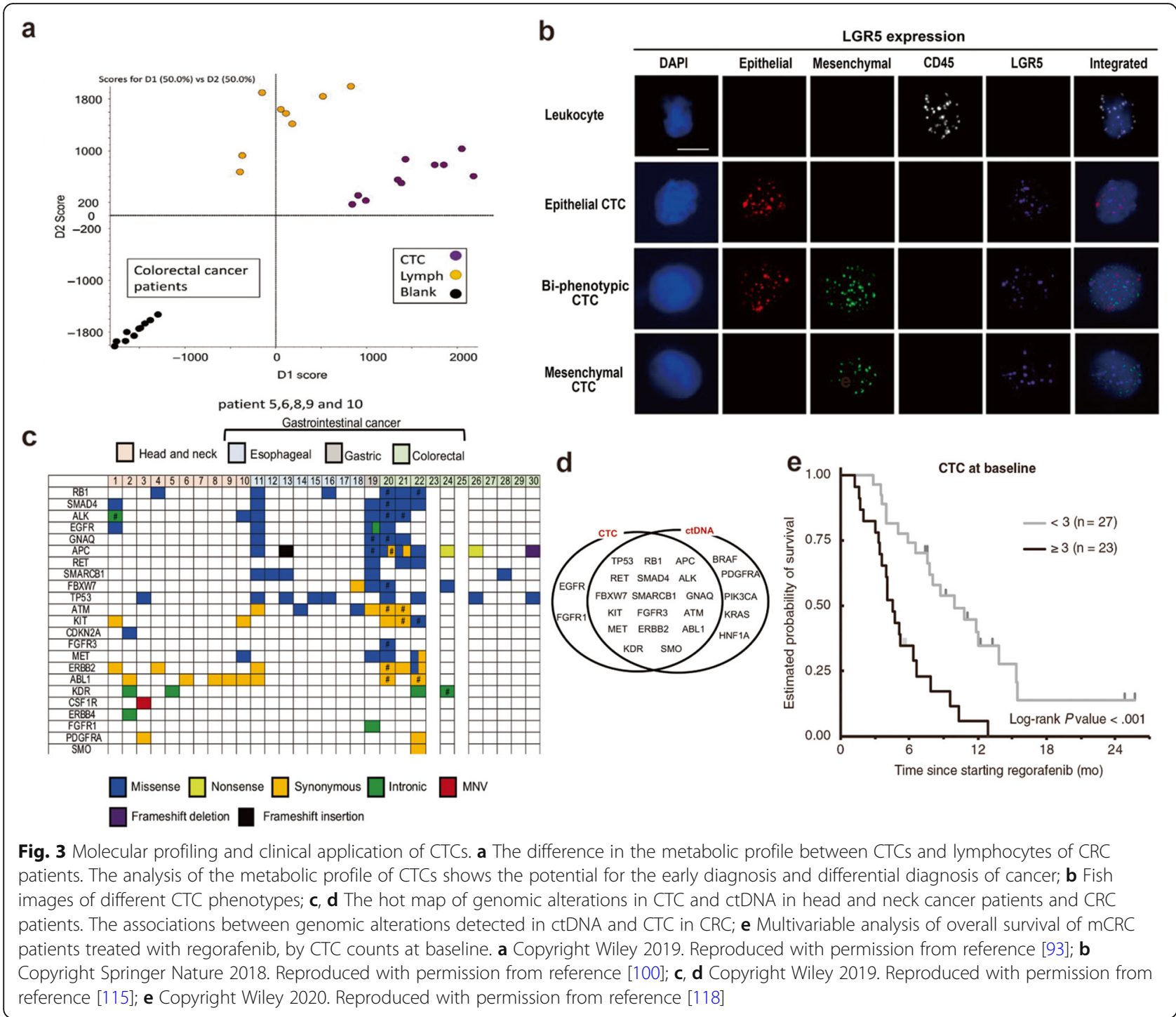

metabolic profile of CTCs has the potential to enable the early diagnosis and differential diagnosis of cancer. Due to the heterogeneity of tumor cells, it is difficult to quantify CTCs accurately according to the biomarkers of the primary tumor. Li et al. quantified the CTCs of $50 \mathrm{CRC}$ patients based on the mRNA levels of six genes (CEA, C-Met, MUC1, CK19, EGFR and EpCAM). The sensitivity of diagnosis was $87 \%$, and the accuracy was $85 \%$ [94]. Compared with enteroscopy, CTC analysis is associated with better patient compliance and a lower economic burden. At present, colonoscopy and biopsy are still the gold standard for diagnosis, and further biopsies can be performed for CTCpositive patients. With further improvements in specificity, CTC detection technologies have the potential to completely replace tissue biopsy as a new diagnostic standard.

In addition to disease diagnosis, some studies have emphasized that CTC load can be used to predict recurrence and metastasis and evaluate patient prognosis.
François et al. analyzed data obtained from the Unicancer Prodige-14 clinical study and found that for CRC patients with potentially resectable liver metastases, high CTC counts $(\geq 3 / 7.5 \mathrm{ml})$ before treatment and 1 month after treatment were an independent prognostic factor for poor OS [95]. The CTC load before and after surgery also plays an important role in predicting recurrence and metastasis [96]. For CRC patients with resected liver metastases, even if the metastasis was completely resected, DFS and OS were often shorter when the preoperative CTC analysis was positive (positive threshold 2/7.5 ml) [97]. Postoperative CTC levels were significantly correlated with preoperative tumor biomarker CA125 levels and recurrence in patients with nonmetastatic CRC $[98,99]$. The ratio of mesenchymal phenotype CTCs to total CTCs can be used to evaluate prognosis and metastasis [100] (Fig. 3b). Although the quantity of CTCs clearly has predictive value, counting 
alone is not sufficient to achieve a comprehensive understanding of the nature and characteristics of tumors. The molecular characteristics of CTCs can provide more accurate prognostic information. Metastasis is the main cause of death in CRC. It is a multistage and complex process, and only cells that meet specific conditions can continue to metastasize. The analysis of CRC tissue samples of different stages has revealed specific chromosomal variations corresponding to each metastatic stage [101]. The chromosome variation in CTCs are consistent with these tissue chromosome variation and corresponding metastasis stage, emphasizing that the molecular characteristics of CTCs has the potential to predict recurrence and metastasis [102]. For example, a mesenchymal phenotype is common in metastatic tumor cells, and patients with CTCs expressing the EMT-associated mRNA Akt-2 have worse prognosis [103]. A study detected the expression of cyclooxygenase-2 (COX-2) in CTCs with different phenotypes in $73 \mathrm{CRC}$ patients. The expression of COX-2 in mesenchymal CTCs was closely related to metastasis [104], indicating the potential for early detection of metastasis and evaluation of prognosis. Nevertheless, the correlation between COX-2 expression in total CTCs and metastasis requires further research.

\section{Guiding precision medicine Quantification}

Patients with advanced CRC are mainly treated with multiline therapy, but the number of suitable patients is limited, and some patients have only one systematic treatment opportunity. Therefore, accurately selecting an effective therapeutic regimen that patients can tolerate is very important to improve prognosis. In addition, acquired drug resistance often occurs during treatment, and there is a certain delay in determining changes in lesions by medical imaging examination. Invasive tissue biopsy is not suitable for frequent use, which makes it difficult to adjust the treatment plan in a timely manner. Several studies have shown that the CTC count reflects tumor burden to some extent and increases with increasing tumor stage. Further investigations on the tumor TNM staging system showed positive correlations between the number detected CTCs and primary tumor size, depth of invasion, lymph node invasion, and distant metastasis, suggesting that CTCs are feasible markers for judging infiltration and metastasis [105]. Anti-EGFR therapy has been confirmed to improve outcome in wild-type RAS CRC. However, there are no recognized biomarkers or molecular markers to predict the prognostic value of systemic therapy. To find a biomarker that can identify patients who cannot benefit from the regimen before treatment begins, Matthew et al. detected CTCs in 42 patients with advanced CRC, and the data obtained from this study were modeled and compared with a larger sample of clinical data (CAIRO2). It was concluded that patients with a high baseline CTC level $(\geq 3 / 7.5 \mathrm{ml})$ were more likely to benefit from intensive therapy [106]. Chou et al. also pointed out that the baseline number of CTCs before chemotherapy was an independent prognostic factor. A prognostic model was established by combining the baseline number of CTCs with other prognostic clinical parameters (past colorectal resection history, Eastern Cooperative Oncology Group (ECOG) score, tumor stage, and histological grade) to assist clinicians in identifying patients who could benefit from palliative treatment prior to the initiation of treatment [107]. Postoperative CTC positivity was identified as an independent prognostic risk factor for recurrence in nonmetastatic CRC patients undergoing radical resection, and 3-year relapse-free survival (RFS) was significantly lower in these patients than in postoperative CTC-negative patients [108]. This study illustrated that a postoperative CTC assessment was necessary to guide risk adjustment and individualized monitoring strategies. Compared with the main clinical tumor biomarkers of CRC (CEA, CA125...), changes in CTC count change are more sensitive and are usually positively correlated with disease progression defined by medical imaging examination. The joint application of these techniques can provide more accurate information and can be used to monitor the response to chemotherapy and disease progression/remission during treatment $[109,110]$.

\section{Single-cell analysis}

CTCs can represent aggressive tumor cell subsets as a whole, and the elimination of these cells is an important measure for preventing recurrence and metastasis. However, the overall assessment of all cells enriched from whole blood may be biased by the influence of lymphocytes. Therefore, precise analysis at single-cell resolution can provide a better understanding of cancer occurrence, development, therapeutic targets and drug resistance.

Whole-genome sequencing CTCs carry the mutation characteristics of the primary tumor. However, the mutations of CTCs measured at different time points show heterogeneity with the mutations of the primary lesion, especially in patients with metastatic tumors. This inconsistency offers effective prognostic information [111]. Patients with metastatic CRC-specific genes expressed in CTCs are often prone to postoperative recurrence, and this information can be used to screen patients in need of postoperative adjuvant therapy. Plastin3 is specifically expressed in metastatic CRC. Preoperative plastin3positive CTCs in peripheral blood are related to poor prognosis, especially in patients with Dukes B and Dukes C [112]. These patients should consider postoperative 
adjuvant chemotherapy. Genome sequencing of CTCs helps to reveal genetic differences between local and metastatic cells.

Currently, anti-EGFR therapy is approved for wildtype RAS CRC. With the emergence of EGFR-targeting drugs such as cetuximab and panizumab, the prognosis of CRC has improved [113]. However KRAS mutation leads to resistance to anti-EGFR therapy, and even patients with wild-type RAS may develop resistance to treatment due to a variety of mechanisms, resulting in a five-year survival rate of only 10 to $15 \%$ [114]. The mechanism of drug resistance may be revealed by analyzing CTC mutations and synthesizing the trend of their mutated gene expression levels over time [115] (Fig. 3c, d). CTCs reflect the real-time status of the tumor genotype, and the molecular profiles of CTCs can help guide treatment plans. Alexios et al. successfully detected KRAS exon 2 mutations in single CTCs from wild-type RAS CRC patients [116]. Yuurin et al. analyzed the mutations in codons 12 and 13 of the KRAS gene in single CTCs and corresponding tumor tissue samples from 7 CRC patients. They found heterogeneity and heterozygosity in KRAS status among the CTCs within a patient and between CTCs and tumor tissues [117]. Regorafenib is a vascular endothelial growth factor receptor (VEGFR) inhibitor, and Satoshi et al. found that the expression of EGFR in CTCs was significantly increased in patients who received regorafenib and were evaluated for progression after treatment compared to baseline CTCs. The authors concluded that increased EGFR expression may be a pattern of resistance and provided supporting evidence for the synergistic effect of regorafenib and anti-EGFR drugs [118] (Fig. 3e). Human epidermal growth factor receptor 2 (HER2) is a member of the EGFR family. Studies have shown that anti-EGFR therapy has poor efficacy in patients with HER2-amplified metastatic CRC, indicating that HER2 amplification can be used as a prognostic biomarker. HER2 is also a potential therapeutic target in CRC. Several clinical studies have shown that anti-HER2 dual-target combination therapy significantly improves the prognosis of HER2positive CRC patients, with an objective response rate reaching $30 \%[119,120]$.

CTCs carry both primary tumor mutations and acquired mutations that differ from the primary tumor. Kong et al. collected preoperative peripheral blood samples and corresponding primary tumor tissue samples from 48 CRC patients and sequenced a gene panel including 39 drug therapy targets and frequently mutated genes. The CTCs were found to carry mutational characteristics similar to those of the primary tumors, and several treatment-related gene mutations (APC, KRAS, TP53, ERBB3, FBXW7 and ERBB2) were detected. They pointed out that the sequencing of single CTCs obtained before surgery and identification of mutations related to targeted therapy may provide information for more timely and accurate treatment interventions [111]. Other emerging therapeutic targets for CRC, including the expression of NrF2/Keap1, NRG1, GARP, are also related to the treatment and prognosis of CRC patients [121123]. The detection and analysis of drug therapy targets on CTCs is of great significance for the development of relevant clinical research.

Immunotherapy is of great significance for patients with advanced refractory CRC, and the main obstacle is how to accurately select the suitable population. PD-L1 expression is an effective indicator for screening lung cancer and melanoma patients who could benefit from immunotherapy; however, the value in CRC is limited. Currently, only CRC patients with microsatellite instability high (MSI-H) can benefit from immunotherapy alone. Approximately $95 \%$ of patients with Lynch syndrome and genetic CRC have tumor microsatellite instability (MSI). This type of tumor has higher immunogenicity and a large number of activated tumorinfiltrating lymphocytes. However only 5 to $8 \%$ of patients have MSI-H tumors. In addition, the tumor mutation burden (TMB) and comprehensive positive score (CPS) of PD-L1 can only provide a certain reference value. POLE and POLD gene mutations are more promising indicators for microsatellite stable (MSS) CRC [124]. Stenzinger et al. detected somatic POLE mutations in up to $12.3 \%$ of MSS CRC patients [125]. In addition, for MSS patients, those with POLE/POLD mutations showed high TMB and higher PD-L1/PD-1 gene expression levels. Overall, the detection of POLE/POLD mutations is meaningful in guiding immunotherapy for CRC, especially for MSS CRC patients [126-128].

Transcriptome analysis Transcriptome analysis has been widely used to detect gene expression levels (mRNA) in tumor cell populations to understand the heterogeneity between tumors. Classifying tumors into subgroups using different molecules can help researchers predict the treatment response and prognosis of patients. However, transcriptome analysis of cell populations cannot reveal intratumoral heterogeneity. Therefore, to comprehensively understand intratumoral and intertumoral heterogeneity, transcriptome analysis at single-cell resolution has attracted extensive attention. The advantages of real-time detection and the better response to heterogeneity make CTCs a suitable object for singlecell transcriptome analysis [129].

Cells expressing leucine-rich repeat-containing gprotein coupled receptor 5 (LGR5) represent the origin of intestinal epithelial tumors. Increased LGR5 expression is closely related to the occurrence and development of a variety of cancers, including $\mathrm{CRC}$, and is associated with 
poor prognosis [130, 131]. Wang et al. enriched the CTCs of 57 CRC patients and evaluated the expression level of LGR5 in each CTC by a multiple-mRNA in situ hybridization (ISH) assay. They concluded that LGR5 expression in CTCs can be used as an indicator to judge metastasis [100]. Yolanda et al. designed a multigene expression panel including GAPDH, VIL1, CLU, TIMP1, TLN1, LOXL3 and ZEB2. CTCs in peripheral blood were captured, and mRNA was extracted from 94 CRC patients. Quantitative real-time PCR was used to quantify mRNA and reflect the expression levels of the abovementioned genes. Prognosis was worse if the expression level of the above genes increased during treatment. After one cycle of treatment ( 4 weeks), patients who cannot benefit from the current regimen could be identified. Compared with computed tomography $(\mathrm{CT})$, evaluation of the treatment response using CTCs is more sensitive and reliable [132]. It is possible to change an ineffective treatment plan as soon as possible, save time spent evaluating the treatment response, and reverse patient outcomes in a timely manner. Li et al. constructed a multiple gene-based algorithm named the six-gene assay, which consists of six CRCrelated genes (CEA, EpCAM, CK19, MUC1, EGFR, and C-Met). CTC quantification was performed for blood samples of 50 patients with recurrent CRC by detecting the mRNA expression levels of the above genes [94]. Compared with the CEA assay, the six-gene assay was more sensitive and accurate in diagnosis and in predicting the 2-year DFS rate. Regorafenib is a multitarget anticancer drug that can effectively improve the survival rate of advanced CRC. However, some patients cannot benefit from it, and the mechanism of primary and acquired drug resistance remains to be studied. Matsusaka et al. performed a clinical study of 50 CRC patients with advanced multiple metastases who were tested for CTCs at baseline, 21 days after the initiation of regorafenib, and after progression. The mRNA levels of EGFR in CTCs were quantified, indicating that the EGFR level at the time of progression was significantly increased compared with that at baseline, suggesting upregulation of EGFR in CTCs may be a mechanism of acquired drug resistance under regorafenib therapy [118].

In addition to single-cell analysis, CTCs can be harvested by culture and then tested for drug sensitivity to select the appropriate drug. One study compared the outcomes of patients who were treated based on a CTC drug sensitivity test (44 patients) with those who were treated based on the traditional method (62 patients). The authors found that the former had significantly longer PFS at 5 months and no drug-related adverse events [133], indicating that CTC drug sensitivity testing is a safe and effective strategy. While a median PFS extension was observed, it did not reach a significant level, and larger cohort studies are needed to demonstrate the potential of this approach.

\section{Conclusion and future development directions}

Decades of research have led to significant advancements in the clinical utility of CTCs in CRC patients. CTCs counting is already FDA-approved for disease monitoring and prognosis assessment in CRC. As a form of liquid biopsy, CTCs also hold great value in facilitating the implementation of precision medicine in CRC. Analyzing CTCs could offer a unique minimally invasive approach to early diagnosis and the characterization and monitoring of dynamic changes in tumor heterogeneity at the genomic, transcriptomic, proteomic and functional levels. However, significant challenges remain before CTCs can be adopted for widespread clinical use in CRC. There is a need to develop and verify detection methods that can achieve high sensitivity and specificity. Among them, nanotechnology is the most active area of research. In addition to technological challenges, thresholds and standards vary between studies, making it difficult to perform a comparative analysis. Therefore, unified standards at different stages are needed. The comprehensive analysis of CTCs and the clinical characteristics of patients will be a powerful tool to promote the development of precision medicine.

\begin{abstract}
Abbreviations
CRC: Colorectal cancer; CTC: Circulating tumor cell; ctDNA: Circulating tumor DNA; miRNA: microRNA; EVs: Extracellular vesicles; EMT: Epithelialmesenchymal transition; MET: Mesenchymal-epithelial transition; EpCAM: Epithelial cell adhesion molecule; FDA: Food and Drug Administration; DAPI: 4',6-diamidino-2-phenylindole; QDs: Quantum dots; PEO: Polyethylene oxide; FRs: Folate receptors; FA: Folic acid;

MACS: Magnetic-activated cell sorting; rVAR2: Recombinant VAR2CSA; STn: Sialyl-Tn; LC: Lung cancer; NSCLC: Non-small cell lung cancer; RCC: Renal cell carcinoma; EGFR: Epidermal growth factor receptor; ECM: Extracellular matrix; AuNPs: Gold nanoparticles; MCF-7: Michigan Cancer Foundation-7; PSA: Prostate-specific antigen; OS: Overall survival; LR: Likelihood ratio; DFS: Disease-free survival; DDFS: Distant disease-free survival; BC: Breast cancer; MBC: Metastatic breast cancer; HR: Hormone receptor; Let: Letrozole; Bev: Bevacizumab; PD-1: Programmed cell death protein-1; PD-

L1: Programmed cell death-ligand 1; AR-V7: Androgen receptor splice variant 7; mCRPC: Metastatic castration-resistant prostate cancer; ALK: Anaplastic lymphoma kinase; NGS: Next-generation sequencing; ASCO: American Society of Clinical Oncology; LSC-MS: Living single-cell mass spectrometry; COX2: Cyclooxygenase-2; ECOG: Eastern Cooperative Oncology Group; FRS: Relapse-free survival; VEGFR: Vascular endothelial growth factor receptor; HER2: Human epidermal growth factor receptor 2; MSI-H: Microsatellite instability high; TMB: Tumor mutation burden; MSS: Microsatellite stable; LGR5: Leucine-rich repeat-containing g-protein coupled receptor 5; ISH: In situ hybridization; CT: Computed tomography; ofCS: Oncofetal chondroitin sulfate; ALL: Acute lymphoblastic leukemia; HCC: Hepatocellular Carcinoma; PAAD: Pancreatic adenocarcinoma; PC: Prostate cancer; GBMLGG: Glioma; mCTC: Mesenchymal CTCs; PCR: Polymerase Chain Reaction; HAl: Hepatic artery infusion
\end{abstract}

\section{Acknowledgements}

Not applicable.

\section{Authors' contributions}

$\mathrm{MJ}$ and $\mathrm{S} L J$ contributed equally to the manuscript. QQH designed this study. $\mathrm{HZ}, \mathrm{QQH}, \mathrm{WXT}$ contributed to the final version of the manuscript. $\mathrm{HZ}, \mathrm{QQH}$, WXT revised the pictures and the manuscript. The author(s) read and approved the final manuscript. 


\section{Funding}

This study was conducted with support from the National Natural Science Foundation of China (No.82000164).

\section{Availability of data and materials}

Not applicable.

\section{Declarations}

Ethics approval and consent to participate

Not applicable.

\section{Consent for publication}

Not applicable.

\section{Competing interests}

The authors declare that they have no competing interests.

\section{Author details}

${ }^{1}$ Department of Oncology, the First Affiliated Hospital of Zhengzhou University, NO.1 Eastern Jianshe Road, Zhengzhou 450052, Henan, China. ${ }^{2}$ BGl College, Zhengzhou University, 40 Daxue Road, Zhengzhou 450052, Henan, China. ${ }^{3}$ Precision Medicine Center, Henan Institute of Medical and Pharmaceutical Sciences, Zhengzhou University, 40 Daxue Road, Zhengzhou 450052, China. ${ }^{4}$ Departments of Otolaryngology, The Second Affiliated Hospital of Zhengzhou University, Zhengzhou 450000, Henan, China. ${ }^{5}$ Academy of medical science, Zhengzhou University, Zhengzhou 450052, Henan, China.

\section{Received: 22 June 2021 Accepted: 27 August 2021}

Published online: 19 November 2021

\section{References}

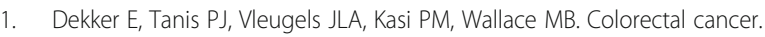
Lancet. 2019;394(10207):1467-80 https://doi.org/10.1016/S0140-6736(1 9)32319-0.

2. Smith R, Andrews K, Brooks D, DeSantis C, Fedewa S, Lortet-Tieulent J, et al. Cancer screening in the United States, 2016: a review of current American Cancer Society guidelines and current issues in cancer screening. CA Cancer J Clin. 2016;66(2):96-114 https://doi.org/10.3322/caac.21336.

3. Punt C, Koopman M, Vermeulen L. From tumour heterogeneity to advances in precision treatment of colorectal cancer. Nat Rev Clin Oncol. 2017;14(4): 235-46 https://doi.org/10.1038/nrclinonc.2016.171.

4. Amin M, Greene F, Edge S, Compton C, Gershenwald J, Brookland R, et al. The eighth edition AJCC Cancer staging manual: continuing to build a bridge from a population-based to a more "personalized" approach to cancer staging. CA Cancer J Clin. 2017;67(2):93-9 https://doi.org/10.3322/caa c.21388

5. Marcuello M, Vymetalkova V, Neves RPL, Duran-Sanchon S, Vedeld HM, Tham E, et al. Circulating biomarkers for early detection and clinical management of colorectal cancer. Mol Asp Med. 2019;69:107-22 https://doi. org/10.1016/j.mam.2019.06.002.

6. Normanno N, Cervantes A, Ciardiello F, De Luca A, Pinto C. The liquid biopsy in the management of colorectal cancer patients: current applications and future scenarios. Cancer Treat Rev. 2018;70:1-8 https://doi. org/10.1016/j.ctrv.2018.07.007

7. Vymetalkova V, Cervena K, Bartu L, Vodicka P. Circulating cell-free DNA and colorectal cancer: a systematic review. Int J Mol Sci. 2018;19(11):3356 https://doi.org/10.3390/ijms19113356.

8. Alix-Panabieres C, Pantel K. Circulating tumor cells: liquid biopsy of cancer. Clin Chem. 2013;59(1):110-8 https://doi.org/10.1373/clinchem.2012.194258.

9. Masuda T, Hayashi N, Iguchi T, Ito S, Eguchi H, Mimori K. Clinical and biological significance of circulating tumor cells in cancer. Mol Oncol. 2016; 10(3):408-17 https://doi.org/10.1016/j.molonc.2016.01.010

10. Jia S, Zhang R, Li Z, Li J. Clinical and biological significance of circulating tumor cells, circulating tumor DNA, and exosomes as biomarkers in colorectal cancer. Oncotarget. 2017:8(33):55632-45 https://doi.org/10.18632/ oncotarget.17184.

11. Suarez-Carmona M, Lesage J, Cataldo D, Gilles C. EMT and inflammation: inseparable actors of cancer progression. Mol Oncol. 2017;11(7):805-23 https://doi.org/10.1002/1878-0261.12095.
12. Li XY, Dong M, Zang XY, Li MY, Zhou JY, Ma JJ, et al. The emerging role of circulating tumor cells in cancer management. Am J Transl Res. 2020;12(2): 332-42.

13. Cohen S, Punt C, lannotti N, Saidman B, Sabbath K, Gabrail N, et al. Relationship of circulating tumor cells to tumor response, progression-free survival, and overall survival in patients with metastatic colorectal cancer. J Clin Oncol. 2008;26(19):3213-21 https://doi.org/10.1200/JCO.2007.15.8923.

14. Konczalla L, Wöstemeier A, Kemper M, Karstens K, Izbicki J, Reeh M. Clinical significance of circulating tumor cells in gastrointestinal carcinomas. Diagnostics (Basel, Switzerland). 2020;10(4):192

15. Zhu Y, Chandra P, Shim Y. Ultrasensitive and selective electrochemical diagnosis of breast cancer based on a hydrazine-au nanoparticle-aptamer bioconjugate. Anal Chem. 2013;85(2):1058-64 https://doi.org/10.1021/ac302 923k

16. Kalikaki A, Politaki H, Souglakos J, Apostolaki S, Papadimitraki E, Georgoulia $\mathrm{N}$, et al. KRAS genotypic changes of circulating tumor cells during treatment of patients with metastatic colorectal cancer. PLoS One. 2014;9(8): e104902 https://doi.org/10.1371/journal.pone.0104902.

17. Yen L, Yeh Y, Chen C, Wang H, Tsai H, Lu C, et al. Detection of KRAS oncogene in peripheral blood as a predictor of the response to cetuximab plus chemotherapy in patients with metastatic colorectal cancer. Clin Cancer Res. 2009;15(13):4508-13 https://doi.org/10.1158/1078-0432.CCR08-3179.

18. Delgado-Urena M, Ortega FG, de Miguel-Perez D, Rodriguez-Martinez A, Garcia-Puche $J$, llyine $H$, et al. Circulating tumor cells criteria (CyCAR) versus standard RECIST criteria for treatment response assessment in metastatic colorectal cancer patients. J Transl Med. 2018;16(1):251 https://doi.org/10.11 86/s12967-018-1624-2.

19. Cabezas-Camarero S, de la Orden GV, Garcia-Barberan V, Mediero-Valeros B, Subhi-Issa Al, Llovet GP, et al. Nasoethmoidal intestinal-type adenocarcinoma treated with Cetuximab: role of liquid biopsy and BEAMing in predicting response to anti-epidermal growth factor receptor therapy. Oncologist. 2019;24(3):293-300 https://doi.org/10.1634/theoncologist.20180387.

20. Yin W, Zhu J, Ma B, Jiang G, Zhu Y, He W, et al. Overcoming obstacles in pathological diagnosis of pulmonary nodules through circulating tumor cell enrichment. Small. 2020;16(25):e2001695 https://doi.org/10.1002/smll.202001 695.

21. Francart M, Lambert J, Vanwynsberghe A, Thompson E, Bourcy M, Polette $M$, et al. Epithelial-mesenchymal plasticity and circulating tumor cells: travel companions to metastases. Dev Dyn. 2018:247(3):432-50 https://doi.org/10.1 002/dvdy.24506

22. Riethdorf $\mathrm{S}$, Fritsche $\mathrm{H}$, Müller $\mathrm{V}$, et al. Detection of circulating tumor cells in peripheral blood of patients with metastatic breast cancer: a validation study of the CellSearch system. Clin Cancer Res. 2007:13(3):920-8.

23. Cui H, Li R, Du J, et al. Rapid and efficient isolation and detection of circulating tumor cells based on $\mathrm{ZnS}: \mathrm{Mn}(2+)$ quantum dots and magnetic nanocomposites. Talanta. 2019;202:230-6.

24. Lee AW, Lin FX, Wei PL, Jian-Wei G, Chen JK. Binary-blend fibber-based capture assay of circulating tumor cells for clinical diagnosis of colorectal cancer. J Nanobiotechnol. 2018;16(1):4.

25. Cui H, Liu Q, Li R, et al. ZnO nanowire-integrated bio-microchips for specific capture and non-destructive release of circulating tumor cells. Nanoscale. 2020;12(3):1455-63.

26. Zhu DM, Wu L, Suo M, et al. Engineered red blood cells for capturing circulating tumor cells with high performance. Nanoscale. 2018;10(13):6014-23.

27. Chen M, Liu A, Chen B, et al. Erythrocyte-derived vesicles for circulating tumor cell capture and specific tumor imaging. Nanoscale. 2019;11(25): 12388-96.

28. Soleymani J, Hasanzadeh M, Shadjou N, Somi MH, Jouyban A. Spectrofluorimetric cytosensing of colorectal cancer cells using terbium-doped dendritic fibrous nano-silica functionalized by folic acid: a novel optical cytosensor for cancer detection. J Pharm Biomed Anal. 2020;180:113077.

29. Salanti A, Clausen TM, Agerbaek MO, et al. Targeting human cancer by a glycosaminoglycan binding malaria protein. Cancer Cell. 2015;28(4):500-14.

30. Agerbaek MO, Bang-Christensen SR, Yang MH, et al. The VAR2CSA malaria protein efficiently retrieves circulating tumor cells in an EpCAMindependent manner. Nat Commun. 2018:9(1):3279.

31. Bang-Christensen SR, Pedersen RS, Pereira MA, et al. Capture and detection of circulating glioma cells using the recombinant VAR2CSA malaria protein. Cells. 2019;8(9):998. 
32. Agerbaek $M \varnothing$, Pereira MA, Clausen TM, et al. Burkitt lymphoma expresses oncofetal chondroitin sulfate without being a reservoir for placental malaria sequestration. Int J Cancer. 2017;140(7):1597-608.

33. Agerbaek MO, Bang-Christensen S, Salanti A. Fighting cancer using an Oncofetal glycosaminoglycan-binding protein from malaria parasites. Trends Parasitol. 2019;35(3):178-81.

34. Zhao K, Cheng G, Zhang B, et al. Targeting delivery of partial VAR2CSA peptide guided N-2-Hydroxypropyl trimethyl ammonium chloride chitosan nanoparticles for multiple cancer types. Mater Sci Eng C Mater Biol Appl. 2020;106:110171.

35. Sand NT, Petersen TB, Bang-Christensen SR, et al. Optimization of rVAR2based isolation of cancer cells in blood for building a robust assay for clinical detection of circulating tumor cells. Int J Mol Sci. 2020;21(7):2401.

36. Neves $M$, Azevedo R, Lima $L$, et al. Exploring sialyl-Tn expression in microfluidic-isolated circulating tumour cells: a novel biomarker and an analytical tool for precision oncology applications. New Biotechnol. 2019;49: 77-87.

37. Scharpenseel H, Hanssen A, Loges S, et al. EGFR and HER3 expression in circulating tumor cells and tumor tissue from non-small cell lung cancer patients. Sci Rep. 2019;9(1):7406.

38. Huang Q, Wang FB, Yuan $\mathrm{CH}$, et al. Gelatin nanoparticle-coated silicon beads for density-selective capture and release of heterogeneous circulating tumor cells with high purity. Theranostics. 2018;8(6):1624-35.

39. Ma S, Zhou X, Chen Q, et al. Multi-targeting magnetic hyaluronan capsules efficiently capturing circulating tumor cells. J Colloid Interface Sci. 2019;545: 94-103.

40. Bu J, Nair A, Kubiatowicz $L$, et al. Surface engineering for efficient capture of circulating tumor cells in renal cell carcinoma: from nanoscale analysis to clinical application. Biosens Bioelectron. 2020;162:112250.

41. Beck TN, Boumber YA, Aggarwal C, et al. Circulating tumor cell and cell-free RNA capture and expression analysis identify platelet-associated genes in metastatic lung cancer. BMC Cancer. 2019;19(1):603.

42. Tian $X$, Sha $X$, Feng $Y$, et al. A magnetic dynamic microbiointerface with biofeedback mechanism for cancer cell capture and release. ACS App Mater Interfaces. 2019;11(44):41019-29.

43. Miao P, Tang Y. Gold nanoparticles-based multipedal DNA Walker for ratiometric detection of circulating tumor cell. Anal Chem. 2019;91(23): 15187-92.

44. Gorges TM, Penkalla N, Schalk T, et al. Enumeration and molecular characterization of tumor cells in lung cancer patients using a novel in vivo device for capturing circulating tumor cells. Clin Cancer Res. 2016;22(9): 2197-206.

45. Wei D, Zeng X, Yang Z, et al. Visualizing interactions of circulating tumor cell and dendritic cell in the blood circulation using in vivo imaging flow cytometry. IEEE Trans Biomed Eng. 2019;66(9):2521-6.

46. Tang M, Xia HF, Xu CM, et al. Magnetic chip based extracorporeal circulation: a new tool for circulating tumor cell in vivo detection. Anal Chem. 2019;91(23):15260-6.

47. Li C, Pan R, Li P, et al. Hydrogen peroxide-responsive nanoprobe assists circulating tumor cell identification and colorectal cancer diagnosis. Anal Chem. 2017;89(11):5966-75.

48. Hattori M, Nakanishi H, Yoshimura M, et al. Circulating tumor cells detection in tumor draining vein of breast cancer patients. Sci Rep. 2019;9(1):18195.

49. Kaifi JT, Kunkel M, Das A, et al. Circulating tumor cell isolation during resection of colorectal cancer lung and liver metastases: a prospective trial with different detection techniques. Cancer Biol Ther. 2015;16(5): 699-708.

50. Tsutsuyama M, Nakanishi $H$, Yoshimura M, et al. Detection of circulating tumor cells in drainage venous blood from colorectal cancer patients using a new filtration and cytology-based automated platform. PLoS One. 2019; 14(2):e0212221.

51. Harouaka R, Nisic M, Zheng S. Circulating tumor cell enrichment based on physical properties. J Lab Autom. 2013;18(6):455-68.

52. Hou HW, Warkiani ME, Khoo BL, et al. Isolation and retrieval of circulating tumor cells using centrifugal forces. Sci Rep. 2013;3:1259.

53. Yusa A, Toneri M, Masuda T, et al. Development of a new rapid isolation device for circulating tumor cells (CTCS) using 3D palladium filter and its application for genetic analysis. PLoS One. 2014;9(2):e88821.

54. Oh BY, Kim J, Lee WY, Kim HC. A new size-based platform for circulating tumor cell detection in colorectal cancer patients. Clin Colorectal Cancer. 2017; 16(3):214-9
55. Pulikkathodi AK, Sarangadharan I, Chen YH, et al. Dynamic monitoring of transmembrane potential changes: a study of ion channels using an electrical double layer-gated FET biosensor. Lab Chip. 2018;18(7):1047-56.

56. Ghassemi P, Ren X, Foster BM, Kerr BA, Agah M. Post-enrichment circulating tumor cell detection and enumeration via deformability impedance cytometry. Biosens Bioelectron. 2020;150:111868.

57. Campton D, Ramirez A, Nordberg J, et al. High-recovery visual identification and single-cell retrieval of circulating tumor cells for genomic analysis using a dual-technology platform integrated with automated immunofluorescence staining. BMC Cancer. 2015;15:360.

58. Jiang J, Zhao H, Shu W, et al. An integrated microfluidic device for rapid and high-sensitivity analysis of circulating tumor cells. Sci Rep. 2017;7:42612.

59. Xu M, Zhao H, Chen J, et al. An integrated microfluidic chip and its clinical application for circulating tumor cell isolation and single-cell analysis. Cytometry A. 2020;97(1):46-53.

60. Johnson ES, Anand RK, Chiu DT. Improved detection by ensemble-decision aliquot ranking of circulating tumor cells with low numbers of a targeted surface antigen. Anal Chem. 2015;87(18):9389-95.

61. Johnson ES, Xu S, Yu HM, et al. Isolating rare cells and circulating tumor cells with high purity by sequential eDAR. Anal Chem. 2019;91(22):1460510.

62. Chen J, Liu CY, Wang X, Sweet E, Liu N, Gong X, et al. 3D printed microfluidic devices for circulating tumor cells (CTCS) isolation. Biosens Bioelectron. 2020;150:111900 https://doi.org/10.1016/j.bios.2019.111900.

63. Goldkorn A, Ely B, Quinn DI, Tangen CM, Fink LM, Xu T, et al. Circulating tumor cell counts are prognostic of overall survival in SWOG S0421: a phase III trial of docetaxel with or without atrasentan for metastatic castrationresistant prostate cancer. J Clin Oncol. 2014;32(11):1136-42 https://doi.org/1 0.1200/JCO.2013.51.7417.

64. Satelli A, Batth I, Brownlee Z, Mitra A, Zhou S, Noh H, et al. EMT circulating tumor cells detected by cell-surface vimentin are associated with prostate cancer progression. Oncotarget. 2017:8(30):49329-37 https://doi.org/10.1 8632/oncotarget.17632.

65. Miyamoto DT, Lee RJ, Kalinich M, LiCausi JA, Zheng Y, Chen T, et al. An RNA-based digital circulating tumor cell signature is predictive of drug response and early dissemination in prostate cancer. Cancer Discov. 2018; 8(3):288-303 https://doi.org/10.1158/2159-8290.CD-16-1406.

66. Bidard F-C, Peeters DJ, Fehm T, Nolé F, Gisbert-Criado R, Mavroudis D, et al. Clinical validity of circulating tumour cells in patients with metastatic breast cancer: a pooled analysis of individual patient data. Lancet Oncol. 2014; 15(4):406-14 https://doi.org/10.1016/S1470-2045(14)70069-5.

67. Goodman CR, Seagle BL, Friedl TWP, Rack B, Lato K, Fink V, et al. Association of circulating tumor cell status with benefit of radiotherapy and survival in early-stage breast cancer. JAMA Oncol. 2018;4(8):e180163 https://doi.org/1 0.1001/jamaoncol.2018.0163.

68. Radovich M, Jiang G, Hancock BA, Chitambar C, Nanda R, Falkson C, et al. Association of Circulating Tumor DNA and circulating tumor cells after neoadjuvant chemotherapy with disease recurrence in patients with triplenegative breast Cancer: preplanned secondary analysis of the BRE12-158 randomized clinical trial. JAMA Oncol. 2020;6(9):1410-5 https://doi.org/10.1 001/jamaoncol.2020.2295.

69. Guan X, Ma F, Li C, Wu S, Hu S, Huang J, et al. The prognostic and therapeutic implications of circulating tumor cell phenotype detection based on epithelial-mesenchymal transition markers in the first-line chemotherapy of HER2-negative metastatic breast cancer. Cancer Commun (Lond). 2019;39(1):1 https://doi.org/10.1186/s40880-018-0346-4.

70. Magbanua MJM, Savenkov O, Asmus EJ, Ballman KV, Scott JH, Park JW, et al. Clinical significance of circulating tumor cells in hormone receptor-positive metastatic breast cancer patients who received Letrozole with or without bevacizumab. Clin Cancer Res. 2020;26(18):4911-20 https://doi.org/10.1158/1 078-0432.CCR-20-1329.

71. Chemi F, Rothwell DG, McGranahan N, Gulati S, Abbosh C, Pearce SP, et al. Pulmonary venous circulating tumor cell dissemination before tumor resection and disease relapse. Nat Med. 2019;25(10):1534-9 https://doi.org/1 0.1038/s41591-019-0593-1.

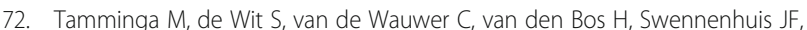
Klinkenberg TJ, et al. Analysis of released circulating tumor cells during surgery for non-small cell lung cancer. Clin Cancer Res. 2020;26(7):1656-66 https://doi.org/10.1158/1078-0432.CCR-19-2541.

73. Frick MA, Feigenberg SJ, Jean-Baptiste SR, Aguarin LA, Mendes A, Chinniah $C$, et al. Circulating tumor cells are associated with recurrent disease in 
patients with early-stage non-small cell lung cancer treated with stereotactic body radiotherapy. Clin Cancer Res. 2020;26(10):2372-80 https:// doi.org/10.1158/1078-0432.CCR-19-2158.

74. Cheng Y, Luo L, Zhang J, Zhou M, Tang Y, He G, et al. Diagnostic value of different phenotype circulating tumor cells in hepatocellular carcinoma. J Gastrointest Surg. 2019;23(12):2354-61 https://doi.org/10.1007/s11605-01804067-y.

75. Ha Y, Kim TH, Shim JE, Yoon S, Jun MJ, Cho YH, et al. Circulating tumor cells are associated with poor outcomes in early-stage hepatocellular carcinoma: a prospective study. Hepatol Int. 2019;13(6):726-35 https://doi.org/10.1007/ s12072-019-09994-9.

76. Hamid F, Gopalan V, Matos M, Lu C, Lam A. Genetic heterogeneity of single circulating tumour cells in colorectal carcinoma. Int J Mol Sci. 2020;21(20): 7766 https://doi.org/10.3390/ijms21207766.

77. Mostert B, Sieuwerts AM, Kraan J, Bolt-de Vries J, van der Spoel P, van Galen $A$, et al. Gene expression profiles in circulating tumor cells to predict prognosis in metastatic breast cancer patients. Ann Oncol. 2015;26(3):510-6 https://doi.org/10.1093/annonc/mdu557.

78. Malihi PD, Graf RP, Rodriguez A, Ramesh N, Lee J, Sutton R, et al. Single-cell circulating tumor cell analysis reveals genomic instability as a distinctive feature of aggressive prostate cancer. Clin Cancer Res. 2020;26(15):4143-53 https://doi.org/10.1158/1078-0432.CCR-19-4100.

79. Khattak MA, Reid A, Freeman J, Pereira M, McEvoy A, Lo J, et al. PD-L1 expression on circulating tumor cells may be predictive of response to Pembrolizumab in advanced melanoma: results from a pilot study. Oncologist. 2020;25(3):e520-e27 https://doi.org/10.1634/theoncologist.20190557.

80. Liu M, Wang R, Sun X, Liu Y, Wang Z, Yan J, et al. Prognostic significance of PD-L1 expression on cell-surface vimentin-positive circulating tumor cells in gastric cancer patients. Mol Oncol. 2020;14(4):865-81 https://doi.org/10.1 002/1878-0261.12643.

81. Papadaki MA, Koutsopoulos AV, Tsoulfas PG, Lagoudaki E, Aggouraki D, Monastirioti A, et al. Clinical relevance of immune checkpoints on circulating tumor cells in breast cancer. Cancers (Basel). 2020;12(2):376 https://doi.org/10.3390/cancers12020376.

82. Ilie M, Szafer-Glusman E, Hofman V, Chamorey E, Lalvee S, Selva E, et al. Detection of PD-L1 in circulating tumor cells and white blood cells from patients with advanced non-small-cell lung cancer. Ann Oncol. 2018;29(1): 193-9 https://doi.org/10.1093/annonc/mdx636.

83. Koh Y, Yagi S, Akamatsu H, Kanai K, Hayata A, Tokudome N, et al. Heterogeneous expression of programmed death receptor-ligand 1 on circulating tumor cells in patients with lung cancer. Clin Lung Cancer. 2019; 20(4):270-7 e1 https://doi.org/10.1016/j.cllc.2019.03.004.

84. Scher HI, Lu D, Schreiber NA, Louw J, Graf RP, Vargas HA, et al. Association of AR-V7 on circulating tumor cells as a treatment-specific biomarker with outcomes and survival in castration-resistant prostate cancer. JAMA Oncol. 2016;2(11):1441-9 https://doi.org/10.1001/jamaoncol.2016.1828.

85. Nakasato T, Kusaka C, Ota M, Hasebe Y, Ueda K, Unoki T, et al. A novel, circulating tumor cell enrichment method reduces ARv7 false positivity in patients with castration-resistant prostate cancer. Diagnostics (Basel). 2020; 10(3):151 https://doi.org/10.3390/diagnostics10030151.

86. Pailler E, Faugeroux V, Oulhen M, Mezquita L, Laporte M, Honore A, et al. Acquired resistance mutations to ALK inhibitors identified by single circulating tumor cell sequencing in ALK-rearranged non-small-cell lung cancer. Clin Cancer Res. 2019;25(22):6671-82 https://doi.org/10.1158/10780432.CCR-19-1176.

87. Chen W, Zhang J, Huang L, Chen L, Zhou Y, Tang D, et al. Detection of HER2-positive circulating tumor cells using the LiquidBiopsy system in breast cancer. Clin Breast Cancer. 2019;19(1):e239-e46 https://doi.org/10.101 6/j.clbc.2018.10.009.

88. Gerdtsson AS, Thiele JA, Shishido SN, Zheng S, Schaffer R, Bethel K, et al. Single cell correlation analysis of liquid and solid biopsies in metastatic colorectal cancer. Oncotarget. 2019;10(66):7016-30 https://doi.org/10.18632/ oncotarget.27271.

89. Wang Q, Zhao L, Han L, Tuo X, Ma S, Wang Y, et al. The discordance of gene mutations between circulating tumor cells and primary/metastatic tumor. Mol Ther Oncolytics. 2019;15:21-9 https://doi.org/10.1016/j.omto.201 9.08.006.

90. D'Oronzo S, Lovero D, Palmirotta R, Stucci LS, Tucci M, Felici C, et al. Dissection of major cancer gene variants in subsets of circulating tumor cells in advanced breast cancer. Sci Rep. 2019;9(1):17276 https://doi.org/10.1 038/s41598-019-53660-x.

91. Yang C, Zhuang W, Hu Y, Zhu L. Clinical significance of peripheral circulating tumor cell counts in colorectal polyps and non-metastatic colorectal cancer. World J Surg Oncol. 2018;16(1):13.

92. Tsai W-S, Nimgaonkar A, Segurado O, et al. Prospective clinical study of circulating tumor cells for colorectal cancer screening. J Clin Oncol. 2018; 36(4_suppl):556.

93. Abouleila Y, Onidani K, Ali A, et al. Live single cell mass spectrometry reveals cancer-specific metabolic profiles of circulating tumor cells. Cancer Sci. 2019;110(2):697-706

94. Shou X, Li Y, Hu W, et al. Six-gene assay as a new biomarker in the blood of patients with colorectal cancer: establishment and clinical validation. Mol Oncol. 2019;13(4):781-91.

95. Bidard F-C, Kiavue N, Ychou M, et al. Circulating tumor cells and circulating tumor DNA detection in potentially resectable metastatic colorectal cancer: a prospective ancillary study to the unicancer prodige-14 trial. Cells. 2019; 8(6):516.

96. Hinz S, Hendricks A, Wittig A, et al. Detection of circulating tumor cells with CK20 RT-PCR is an independent negative prognostic marker in colon cancer patients - a prospective study. BMC Cancer. 2017;17(1):53.

97. Arrazubi V, Mata E, Antelo ML, et al. Circulating tumor cells in patients undergoing resection of colorectal cancer liver metastases. Clinical utility for long-term outcome: a prospective trial. Ann Surg Oncol. 2019;26(9):2805-11.

98. Yang C, Zou K, Zheng L, Xiong B. Prognostic and clinicopathological significance of circulating tumor cells detected by RT-PCR in non-metastatic colorectal cancer: a meta-analysis and systematic review. BMC Cancer. 2017; 17(1):725.

99. Wang $D$, Yang $Y$, Jin $L$, et al. Prognostic models based on postoperative circulating tumor cells can predict poor tumor recurrence-free survival in patients with stage II-III colorectal cancer. J Cancer. 2019;10(19):4552-63.

100. Wang W, Wan L, Wu S, et al. Mesenchymal marker and LGR5 expression levels in circulating tumor cells correlate with colorectal cancer prognosis. Cell Oncol. 2018:41(5):495-504.

101. Cayrefourcq L, Mazard T, Joosse S, et al. Establishment and characterization of a cell line from human circulating colon cancer cells. Cancer Res. 2015; 75(5):892-901.

102. Joosse SA, Souche FR, Babayan A, et al. Chromosomal aberrations associated with sequential steps of the metastatic cascade in colorectal cancer patients. Clin Chem. 2018;64(10):1505-12.

103. Ning Y, Zhang W, Hanna DL, et al. Clinical relevance of EMT and stem-like gene expression in circulating tumor cells of metastatic colorectal cancer patients. Pharm J. 2018;18(1):29-34.

104. Cai J, Huang L, Huang J, et al. Associations between the cyclooxygenase-2 expression in circulating tumor cells and the clinicopathological features of patients with colorectal cancer. J Cell Biochem. 2019;120(4):4935-41.

105. Szczerba BM, Castro-Giner F, Vetter M, et al. Neutrophils escort circulating tumour cells to enable cell cycle progression. Nature. 2019;566(7745):553-7.

106. Krebs MG, Renehan AG, Backen A, et al. Circulating tumor cell enumeration in a phase II trial of a four-drug regimen in advanced colorectal cancer. Clin Colorectal Cancer. 2015;14(2):115-122.e111-112.

107. Chou WC, Wu MH, Chang PH, et al. A prognostic model based on circulating tumour cells is useful for identifying the poorest survival outcome in patients with metastatic colorectal cancer. Int J Biol Sci. 2018; 14(2):137-46.

108. Yang C, Shi D, Wang S, Wei C, Zhang C, Xiong B. Prognostic value of preand post-operative circulating tumor cells detection in colorectal cancer patients treated with curative resection: a prospective cohort study based on ISET device. Cancer Manag Res. 2018;10:4135-44.

109. Tan K, Leong SM, Kee Z, et al. Longitudinal monitoring reveals dynamic changes in circulating tumor cells (CTCs) and CTC-associated miRNAs in response to chemotherapy in metastatic colorectal cancer patients. Cancer Lett. 2018;423:1-8.

110. Sastre J, Orden V, Martinez A, et al. Association between baseline circulating tumor cells, molecular tumor profiling, and clinical characteristics in a large cohort of chemo-naive metastatic colorectal cancer patients prospectively collected. Clin Colorectal Cancer. 2020;19(3):e110-6.

111. Kong S, Liu X, Suhaimi N, et al. Molecular characterization of circulating colorectal tumor cells defines genetic signatures for individualized cancer care. Oncotarget. 2017:8(40):68026-37. 
112. Yokobori $T$, linuma $H$, Shimamura $T$, et al. Plastin3 is a novel marker for circulating tumor cells undergoing the epithelial-mesenchymal transition and is associated with colorectal cancer prognosis. Cancer Res. 2013;73(7): 2059-69.

113. Miyamoto $Y$, Suyama K, Baba H. Recent advances in targeting the EGFR signaling pathway for the treatment of metastatic colorectal cancer. Int J Mol Sci. 2017;18(4):752

114. Arnold M, Sierra MS, Laversanne M, Soerjomataram I, Jemal A, Bray F. Global patterns and trends in colorectal cancer incidence and mortality. Gut. 2017; 66(4):683-91.

115. Onidani K, Shoji H, Kakizaki T, et al. Monitoring of cancer patients via nextgeneration sequencing of patient-derived circulating tumor cells and tumor DNA. Cancer Sci. 2019;110(8):2590-9.

116. Matikas A, Voutsina A, Lagoudaki $E$, et al. Detection of KRAS exon 2 mutations in circulating tumor cells isolated by the ISET system from patients with RAS wild type metastatic colorectal cancer. Transl Oncol. 2017; 10(4):693-8.

117. Kondo Y, Hayashi K, Kawakami K, Miwa Y, Hayashi H, Yamamoto M. KRAS mutation analysis of single circulating tumor cells from patients with metastatic colorectal cancer. BMC Cancer. 2017;17(1):311.

118. Matsusaka S, Hanna DL, Ning Y, et al. Epidermal growth factor receptor mRNA expression: a potential molecular escape mechanism from regorafenib. Cancer Sci. 2020;111(2):441-50.

119. La Salvia A, Lopez-Gomez V, Garcia-Carbonero R. HER2-targeted therapy: an emerging strategy in advanced colorectal cancer. Expert Opin Investig Drugs. 2019;28(1):29-38.

120. Meric-Bernstam F, Hurwitz H, Raghav KPS, et al. Pertuzumab plus trastuzumab for HER2-amplified metastatic colorectal cancer (MyPathway): an updated report from a multicentre, open-label, phase 2a, multiple basket study. Lancet Oncol. 2019;20(4):518-30.

121. Galan-Cobo A, Sitthideatphaiboon P, Qu X, et al. KRASLKB1 and KEAP1/NRF2 pathways cooperatively promote metabolic reprogramming with enhanced glutamine dependence in -mutant lung adenocarcinoma. Cancer Res. 2019; 79(13):3251-67.

122. Stahler A, Heinemann $V$, Neumann J, et al. Prevalence and influence on outcome of HER2/neu, HER3 and NRG1 expression in patients with metastatic colorectal cancer. Anti-Cancer Drugs. 2017;28(7):717-22.

123. Salem M, Wallace $C$, Velegraki $M$, et al. GARP dampens cancer immunity by sustaining function and accumulation of regulatory $T$ cells in the colon. Cancer Res. 2019;79(6):1178-90.

124. Angelova M, Charoentong $\mathrm{P}, \mathrm{Hackl} \mathrm{H}$, et al. Characterization of the immunophenotypes and antigenomes of colorectal cancers reveals distinct tumor escape mechanisms and novel targets for immunotherapy. Genome Biol. 2015;16:64.

125. Stenzinger A, Pfarr N, Endris V, et al. Mutations in POLE and survival of colorectal cancer patients--link to disease stage and treatment. Cancer Med. 2014;3(6):1527-38

126. Gong J, Wang C, Lee P, Chu P, Fakih M. POLEResponse to PD-1 blockade in microsatellite stable metastatic colorectal cancer harboring a mutation. J Natl Compr Cancer Netw. 2017;15(2):142-7.

127. Forgó E, Gomez A, Steiner D, Zehnder J, Longacre T. Morphological, immunophenotypical and molecular features of hypermutation in colorectal carcinomas with mutations in DNA polymerase $\varepsilon$ (POLE). Histopathology. 2020;76(3):366-74

128. Palles C, Cazier J, Howarth K, et al. Germline mutations affecting the proofreading domains of POLE and POLD1 predispose to colorectal adenomas and carcinomas. Nat Genet. 2013;45(2):136-44.

129. Mostert B, Sieuwerts AM, Bolt-de Vries J, et al. mRNA expression profiles in circulating tumor cells of metastatic colorectal cancer patients. Mol Oncol. 2015;9(4):920-32.

130. Merlos-Suárez A, Barriga F, Jung P, et al. The intestinal stem cell signature identifies colorectal cancer stem cells and predicts disease relapse. Cell Stem Cell. 2011;8(5):511-24.

131. Tanese K, Fukuma M, Yamada T, et al. G-protein-coupled receptor GPR49 is up-regulated in basal cell carcinoma and promotes cell proliferation and tumor formation. Am J Pathol. 2008;173(3):835-43.

132. Insua YV, Camara J, Vazquez EB, et al. Predicting outcome and therapy response in $\mathrm{mCRC}$ patients using an indirect method for CTCs detection by a multigene expression panel: a multicentric prospective validation study. Int J Mol Sci. 2017:18(6):1265.
133. Guadagni S, Clementi M, Mackay AR, et al. Real-life multidisciplinary treatment for unresectable colorectal cancer liver metastases including hepatic artery infusion with chemo-filtration and liquid biopsy precision oncotherapy: observational cohort study. J Cancer Res Clin Oncol. 2020; 146(5):1273-90

\section{Publisher's Note}

Springer Nature remains neutral with regard to jurisdictional claims in published maps and institutional affiliations.
Ready to submit your research? Choose BMC and benefit from:

- fast, convenient online submission

- thorough peer review by experienced researchers in your field

- rapid publication on acceptance

- support for research data, including large and complex data types

- gold Open Access which fosters wider collaboration and increased citations

- maximum visibility for your research: over $100 \mathrm{M}$ website views per year

At $\mathrm{BMC}$, research is always in progress.

Learn more biomedcentral.com/submissions 